Rev. Inst. Flor. v. 28 n. 2 p. $135-157$ dez. 2016

http://dx.doi.org/10.24278/2178-5031.201628205

ISSN impresso 0103-2674/on-line 2178-5031

\title{
AVES REGISTRADAS EM 14 ÁREAS PROTEGIDAS NO INTERIOR DO ESTADO DE SÃO PAULO, BRASIL ${ }^{1}$ BIRDS RECORDED IN 14 PROTECTED AREAS
OF THE STATE OF SÃO PAULO, SOUTHEASTERN BRAZIL
}

\author{
Alexsander Zamorano ANTUNES ${ }^{2,3}$; Marina Mitsue KANASHIRO²; \\ Marilda Rapp de ESTON ${ }^{2}$
}

\begin{abstract}
RESUMO - Muitas das áreas atualmente protegidas no interior do estado de São Paulo são relativamente pequenas e isoladas de outros remanescentes de vegetação nativa. Considerando que a composição da avifauna pode contribuir para avaliar a relevância dessas áreas para a conservação da biodiversidade no estado de São Paulo, o presente trabalho divulga as espécies de aves encontradas durante levantamentos efetuados em 14 destas localidades no período de 2007 a 2016. Considerando todas as áreas em conjunto foram registradas 339 espécies, 22 ameaçadas de extinção no estado, 53 com distribuição restrita à Mata Atlântica e seis restritas ao Cerrado. As quatro unidades de uso sustentável amostradas apresentaram totais de espécies ameaçadas e de distribuição restrita similares aos obtidos para as 10 unidades de proteção integral (estações ecológicas), o que justificaria a aplicação de uma mesma categoria mais restritiva para todas as 14 áreas.
\end{abstract}

Palavras-chave: espécies ameaçadas; Cerrado; Mata Atlântica; Unidades de Conservação.

\begin{abstract}
Many of the areas currently protected in the state of São Paulo are relatively small and isolated from other remnants of native vegetation. The composition of avifauna can contribute to evaluate the relevance of those areas for biodiversity conservation in the state of São Paulo, so this paper presents bird species found during surveys carried out in 14 of these sites between 2007 and 2016. Considering all areas together we recorded 339 species, 22 threatened in the state, 53 with distribution restricted to the Atlantic Forest and six restricted to Cerrado domain. The four sustainable use units sampled had total of threatened species and restricted distribution similar to those obtained for the 10 full protection units (ecological stations), which would justify the application of the same category for all 14 areas.
\end{abstract}

Keywords: threatened species; Cerrado; Atlantic Forest; Conservation Units.

${ }^{1}$ Recebido para análise em 05.04.2016. Aceito para publicação em 21.11.2016.

${ }^{2}$ Instituto Florestal, Rua do Horto 931, 02377-000 São Paulo, SP, Brasil.

${ }^{3}$ Autor para correspondência: Alexsander Zamorano Antunes - alexza@if.sp.gov.br 


\section{INTRODUÇÃOO}

O Estado de São Paulo foi habitado por povos indígenas por alguns milhares de anos colonizado por europeus a partir do século XVI, porém foram os ciclos econômicos baseados na agricultura para exportação nos séculos XIX e XX que resultaram na perda da maior parte de sua vegetação natural (Dean, 1997). Atualmente, estima-se que restam $43.430 \mathrm{~km}^{2}$ de vegetação natural, o que corresponde a $17,5 \%$ da área do estado (São Paulo, 2016). Especificamente em relação ao dois biomas principais que ocorrem no estado, a Mata Atlântica antes ocupava $69 \%$ da área, estando hoje reduzida a $14 \%\left(23.789,85 \mathrm{~km}^{2}\right)$ enquanto para o Cerrado estes percentuais foram de $14 \%$ para $1 \%, 2.855,55 \mathrm{~km}^{2}$ (Kronka et al., 2005; Fundação SOS Mata Atlântica e Instituto Nacional de Pesquisas Espaciais, 2015). Hoje, apenas 4,5\% do estado $\left(11.205,95 \mathrm{~km}^{2}\right)$ estão no interior de unidades de conservação de proteção integral (Xavier et al., 2008; Fundação Florestal, 2016a Instituto Chico Mendes de Conservação da Biodiversidade, 2016; São Paulo, 2016a).

A maioria das áreas protegidas do interior do estado de São Paulo é relativamente pequena e isolada de outras áreas de vegetação nativa. Muitas também sofreram uso agropecuário intensivo antes de serem adquiridas pelo poder público. Após sua desapropriação, as áreas com vegetação campestre e savânica foram utilizadas pelo Serviço Florestal, posteriormente Instituto Florestal, como Estações Experimentais e Florestas Estaduais principalmente para o cultivo de pinheiros exóticos Pinus spp. e as áreas florestadas como Reservas Florestais, que eventualmente sofriam corte seletivo de árvores. A partir da década de 1980, com a valorizacão das unidades de conservação pela sociedade, algumas dessas áreas, por apresentarem atributo naturais significativos, foram parcial ou integralmente transformadas em Estações Ecológicas, permitindo a regeneração da vegetação nativa uma proteção mais efetiva de sua biodiversidade. Mesmo naquelas áreas utilizadas para a experimentação florestal, em grandes trechos fo permitida a regeneração natural da vegetação, principalmente nas Áreas de Preservação Permanente.
As Estações Experimentais e as Florestas Estaduais são unidades de uso sustentável, enquanto as Estações Ecológicas são de proteção integral, portanto, com objetivos de manejo diferentes. De acordo com a Lei $\mathrm{n}^{\circ} 9.985$, de 18 de julho de 2000, que institui o Sistem Nacional de Unidades de Conservação - SNUC, uma Estação Ecológica tem como objetivo a preservação da natureza e a realização de pesquisas científicas. É de posse e domínio públicos, sendo proibida a visitação pública, exceto com objetivo educacional (Brasil, 2000).

Tendo em vista este histórico, qual será atualmente a relevância destas áreas para conservação da biodiversidade do estado de São Paulo? Considerando que as aves constituem o grupo de organismos mais facilmente inventariado e que a análise das assembleias locais pode oferecer subsídios para responder a esta questão, os objetivos do presente trabalho foram: 1) divulgar as espécies encontradas durante levantamentos efetuados recentemente em algumas destas áreas protegidas e 2) avaliar quais informações obtidas em inventários rápidos não padronizados de aves podem ser utilizadas para a comparação entre áreas.

\section{MATERIAL E MÉTODOS}

Entre 2007 e 2016, por meio de participação em propostas de criação e planos de manejo de unidades de conservação, foram realizados levantamentos rápidos da avifauna em 11 áreas protegidas administradas pelo Instituto Florestal e três administradas, a partir de 2007, pela Fundação Florestal (Bauru, Itaberá e Ribeirão Preto; Figura 1)

Informações sobre as áreas visitadas são apresentadas na Tabela 1 e foram obtidas nas páginas na internet da Fundação Florestal (2016a) e de São Paulo (2016a). Para o número de fitofisionomias foram considerado tanto tipos diferentes, ex. floresta, campo e brejo, quanto fases sucessionais. Essas informações foram obtidas dos planos de manejo das áreas (Fundação Florestal, 2016b; São Paulo, 2016b)

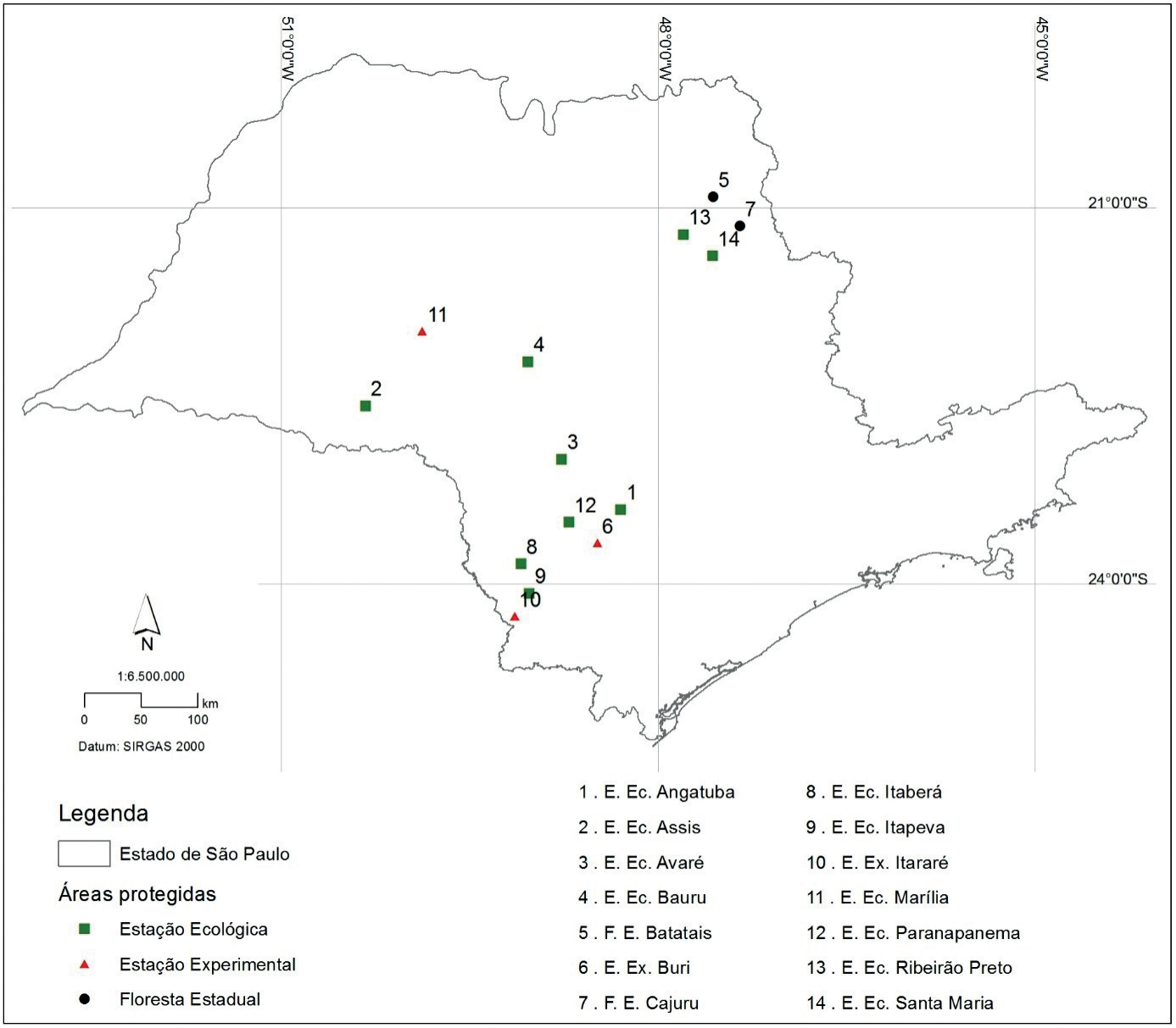

Figura 1. Localização das áreas amostradas no interior do estado de São Paulo.

Figure 1. Location of the sampled areas in the state of São Paulo, southeastern Brazil.

Tabela 1. Áreas amostradas. E. Ec. = Estação Ecológica; E. Ex. = Estação Experimental e F. E. = Floresta Estadual Domínio fitogeográfico predominante: $\mathrm{CE}=$ Cerrado; FES $=$ Floresta Estacional Semidecidual; FOM $=$ Floresta Ombrófila Mista.

Table 1. Sampled areas. E. Ec. $=$ Ecological Station; E. Ex. $=$ Experimental Station and F. E. $=$ State Forest. Phytogeographic predominant domain: $\mathrm{CE}=$ Cerrado; FES $=$ Semideciduous Forest; FOM $=$ Mixed Rain Forest.

\begin{tabular}{ccccccc}
\hline Nome & Área (ha) & Domínio & $\begin{array}{c}\text { Número de } \\
\text { Fitofisionomias }\end{array}$ & $\begin{array}{c}\text { Ano de } \\
\text { Criação }\end{array}$ & Período Amostral & $\begin{array}{c}\text { Esforço } \\
\text { Amostral } \\
\text { (horas) }\end{array}$ \\
\hline E. Ec. Angatuba & $1.394,15$ & FES & 21 & 1985 & 19 a 23/03 e 18 a 21/10/2007 & 50 \\
E. Ec. Assis & $1.760,64$ & CE & 15 & 1992 & 01 a 05/10/2007 & 38 \\
E. Ec. Avaré & 709,02 & CE & 12 & 2010 & 02 a 03/03/2010 & 10 \\
E. Ec. Bauru & 287,98 & FES & 18 & 1987 & 17 a 21/09 e 19 a 22/11/2007 & 50
\end{tabular}




\begin{tabular}{|c|c|c|c|c|c|c|}
\hline Nome & Área (ha) & Domínio & $\begin{array}{l}\text { Número de } \\
\text { Fitofisionomias }\end{array}$ & $\begin{array}{l}\text { Ano de } \\
\text { Criação }\end{array}$ & Período Amostral & $\begin{array}{l}\text { Esforço } \\
\text { Amostral } \\
\text { (horas) }\end{array}$ \\
\hline F. E. Batatais & $1.478,55$ & $\mathrm{CE}$ & $10^{*}$ & 1943 & 08 a $10 / 12 / 2014$ & 16 \\
\hline E. Ex. Buri & $1.080,68$ & FES & 14 & 1960 & 20 a $21 / 10 / 2014$ & 15 \\
\hline F. E. Cajuru & $1.909,56$ & $\mathrm{CE}$ & 16 & 1962 & 28 a $30 / 10 / 2013$ & 25 \\
\hline E. Ec. Itaberá & 180 & FOM & 9 & 1987 & 17 e $18 / 09 / 2008$ & 18 \\
\hline E. Ec. Itapeva & 106,77 & $\mathrm{CE}$ & 11 & 1985 & 15 a $19 / 09 / 2008$ & 10 \\
\hline E. Ex. Itararé & $2.379,05$ & FOM & 9* & 1960 & $\begin{array}{c}22 \text { a } 24 / 10 / 2014 \text { e } 08 \text { e } \\
09 / 03 / 2016\end{array}$ & 31 \\
\hline E. Ec. Marília & 607,14 & FES & 14 & 2010 & 22 a $24 / 01 / 2013$ & 21 \\
\hline E. Ec. Paranapanema & 635,20 & FES & 15 & 1993 & 17 a $20 / 03 / 2014$ & 16 \\
\hline E. Ec. Ribeirão Preto & 154,16 & FES & 10 & 1984 & 16 a 19/03/2009 & 13 \\
\hline E. Ec. Santa Maria & $1.301,35$ & $\mathrm{CE}$ & $13 *$ & 2010 & 04 а $07 / 05 / 2015$ & 22 \\
\hline
\end{tabular}

*Dados da vegetação baseados nos mapeamentos preliminares. *Vegetation data based on preliminary maps.

As aves foram amostradas pelo método de trajetos de distância ilimitada (Willis, 1979; Bibby et al., 1992), que consiste em se deslocar lentamente, perto de $1 \mathrm{~km} / \mathrm{h}$, pelas trilhas e estradas disponíveis, registrando-se cada indivíduo detectado visual e/ou auditivamente. Foram utilizados binóculos 8 x 40, gravador profissional PMD222 Marantz com microfone Sennheiser ME66/K6C para registrar vocalizações e outras manifestações sonoras, e câmera fotográfica digital com zoom ótico de 42x para documentar a presença das espécies. As coordenadas das áreas amostradas foram obtidas com GPS 76CSx Garmin

A nomenclatura científica adotada foi do Comitê Brasileiro de Registros Ornitológicos (Piacentini et al., 2015). Foram consideradas as espécies ameaçadas de extinção no estado de São Paulo (São Paulo, 2014) e as endêmicas ou com distribuição restrita aos Domínios do Cerrado e da Mata Atlântica (Bencke et al., 2006).

As correlações entre as variáveis independentes (área em hectares, número de fitofisionomias locais e esforço amostral) e as variáveis dependentes (riqueza, número de espécies ameaçadas de extinção no estado de São Paulo, espécies com distribuição restrita) foram efetuadas por meio do teste de correlação de postos de Spearman (rs). Valores médios das variáveis dependentes foram comparados através do teste de Mann-Whitney (U). Os testes estatístico foram efetuados com o programa BioEstat 5.0 (Ayres et al., 2010) e os resultados foram considerados significativos para $\mathrm{p}<0,05$ Análise multivariada pelo método "Unweighted Pair Group Method with Arithmetic Mean - UPGMA", distância euclidiana simples, foi utilizada para avaliar a similaridade na avifauna entre as áreas, por meio do programa "MultiVariate Statistical Package - MVSP" (Kovach, 1999).

\section{RESULTADOS E DISCUSSÃO}

Considerando todas as áreas em conjunto, foram registradas 339 espécies de aves, 22 ameaçadas de extinção, 53 com distribuição restrita à Mata Atlântica e seis restritas ao Cerrado (Tabelas 2 e 3, Figuras 2 e 3). Duzentas e oitenta e duas espécies (83\%) foran fotografadas ou gravadas. Willis e Onik (1981, 2003) efetuaram levantamentos rápidos na década de 1980 em todas essas áreas, com exceção de Itaberá e Paranapanema, encontrando espécies não registradas no presente estudo.
Optou-se por não incluir esses dados pois, mesmo sendo áreas protegidas, tratam-se de emanescentes relativamente pequenos clados de outras áreas de vegetaça na nava, (1) (a) Apencs locails ao longo do tenpo (Anthes, 2005). Apenas outras sete áreas protegidas no nterior do estado contam com inventário recentes de avifauna publicados (Dias, 2000; Pozza e Pires, 2003; Develey et al., 2005; Motta-Júnior et al., 2008; Cavarzere et al. 2009; Telles e Dias, 2010; Fieker et al., 2013; Lucindo et al., 2015).

Tabela 2. Espécies registradas com total de deteç̧ões por área. $\mathrm{AM}$ = espécie ameaçada de extinção no estado de São Paulo; $\mathrm{MA}$ = espécie restrita ao Domínio da Mata Atlântica; $\mathrm{CE}$ = espécie restrita ao Domínio do Cerrado; $\mathrm{F}$ = espécie fotografada; $\mathrm{G}=$ vocalização gravada. Áreas Protegidas: Angatuba (An), Assis (As), Avaré (Av), Batatais (Ba), Bauru (Bu), Buri (Br), Cajuru (Ca), Itaberá (It), Itapeva (Ip), Itararé (Ir), Marília (Ma), Paranapanema (Pa) Ribeirão Preto (RP) e Santa Maria (SM).

Table 2. Bird species recorded with detections by area. $\mathrm{AM}=$ threatened species in the state of São Paulo; $\mathrm{MA}=$ species restricted to Atlantic Forest Domain; $\mathrm{CE}=$ species restricted to Cerrado Domain. $\mathrm{F}=$ photographed species; =

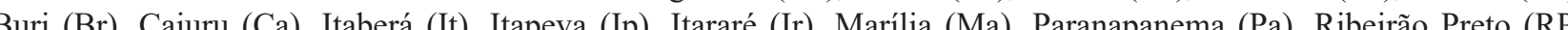
Maria (SM).

\begin{tabular}{|c|c|c|c|c|c|c|c|c|c|c|c|c|c|c|c|}
\hline \multicolumn{3}{|l|}{ Nome do Táxon } & An & As & $\mathrm{Av}$ & $\mathrm{Ba}$ & $\mathrm{Bu}$ & $\mathrm{Br}$ & $\mathrm{Ca}$ & It & Ip & Ir & Ма & $\mathrm{Pa} \mathrm{RF}$ & SM \\
\hline \\
\hline \multicolumn{16}{|l|}{ Tinamidae } \\
\hline Crypturellus obsoletus (Temminck, 1815) & G & & 2 & & & & & & & & 2 & 8 & & & \\
\hline Crypturellus undulatus (Temminck, 1815) & G & AM & & & & & & & 2 & & & & & & \\
\hline Crypturellus parvirostris (Wagler, 1827) & G & & 1 & 12 & 4 & & & & 1 & & 1 & & 4 & & 4 \\
\hline Crypturellus tataupa (Temminck, 1815) & & & & 11 & & & 2 & & & & & & & & \\
\hline Rhynchotus rufescens (Temminck, 1815) & G & AM & & 1 & & & 1 & & 1 & & & 5 & 1 & 1 & \\
\hline Nothura maculosa (Temminck, 1815) & & & & & & & & & & & & & 1 & & \\
\hline \multicolumn{16}{|l|}{ Anseriformes } \\
\hline \multicolumn{16}{|l|}{ Anhimidae } \\
\hline Anhima cornuta (Linnaeus, 1766) & G & AM & & & & & & & & & & & 1 & & \\
\hline \multicolumn{16}{|l|}{ Anatidae } \\
\hline Dendrocygna viduata (Linnaeus, 1766) & $\mathrm{F}$ & & & & & & & & & & & & 1 & 10 & \\
\hline Dendrocygna autumnalis (Linnaeus, 1758) & $\mathrm{F}$ & & & & & & & & & & & & & 6 & \\
\hline Cairina moschata (Linnaeus, 1758) & & & 7 & & & & 4 & & & & 1 & & 1 & 4 & \\
\hline Amazonetta brasiliensis (Gmelin, 1789) & $\mathrm{F}$ & & 1 & & & & 2 & & & & & & & 2 & \\
\hline \multicolumn{16}{|l|}{ Galliformes } \\
\hline \multicolumn{16}{|l|}{ Cracidae } \\
\hline Penelope superciliaris Temminck, 1815 & G & & 24 & 45 & 4 & & 8 & 4 & & 3 & 3 & & & 6 & \\
\hline
\end{tabular}


continuação - Tabela 2

continuation - Table 2

\begin{tabular}{|c|c|c|c|c|c|c|c|c|c|c|c|c|c|c|}
\hline Nome do Táxon & & & An & As & Av $\mathrm{E}$ & $\mathrm{Ba} \quad \mathrm{B}$ & $\mathrm{Bu} \mathrm{Br}$ & $\mathrm{Br} \mathrm{Ca}$ & It & Ip & Ir $\mathrm{N}$ & Ma & $\mathrm{a}$ RP & SM \\
\hline Penelope obscura Temminck, 1815 & $\mathrm{~F}$ & & & & & & & & & & 6 & & & \\
\hline Podicipediformes & & & & & & & & & & & & & & \\
\hline Podicipedidae & & & & & & & & & & & & & & \\
\hline Tachybaptus dominicus (Linnaeus, 1766) & $\mathrm{F}$ & & & & & & & 1 & & & 4 & & & \\
\hline Podilymbus podiceps (Linnaeus, 1758) & $\mathrm{F}$ & & & 1 & & & & 1 & & & & & 1 & \\
\hline Suliformes & & & & & & & & & & & & & & \\
\hline Phalacrocoracidae & & & & & & & & & & & & & & \\
\hline Nannopterum brasilianus (Gmelin, 1789) & $\mathrm{F}$ & & 1 & 1 & & & & & & & & & & \\
\hline Anhingidae & & & & & & & & & & & & & & \\
\hline Anhinga anhinga (Linnaeus, 1766) & $\mathrm{F}$ & & 1 & & & & & & & & & & & \\
\hline Pelecaniformes & & & & & & & & & & & & & & \\
\hline Ardeidae & & & & & & & & & & & & & & \\
\hline Tigrisoma lineatum (Boddaert, 1783) & & & 1 & & & & 1 & & & & & & 1 & \\
\hline Nycticorax nycticorax (Linnaeus, 1758) & $\mathrm{F}$ & & 1 & 1 & & & & & & & & & & \\
\hline Butorides striata (Linnaeus, 1758) & $\mathrm{F}$ & & 2 & & 1 & & & 1 & & & & & 1 & \\
\hline Bubulcus ibis (Linnaeus, 1758) & $\mathrm{F}$ & & 2 & 10 & & & 10 & & & & & & 6 & 6 \\
\hline Ardea alba Linnaeus, 1758 & $\mathrm{~F}$ & & & 1 & & & 1 & 1 & & & & & 1 & 1 \\
\hline Syrigma sibilatrix (Temminck, 1824) & G & & 4 & 1 & 2 & 2 & 4 & 2 & & & & 1 & 2 & \\
\hline Threskiornithidae & & & & & & & & & & & & & & \\
\hline Mesembrinibis cayennensis (Gmelin, 1789) & F G & & 2 & & & 2 & 2 & 1 & & & & 1 & 3 & \\
\hline Phimosus infuscatus (Lichtenstein, 1823) & $\mathrm{F}$ & & & 1 & & & & & & & & 1 & & \\
\hline Theristicus caudatus (Boddaert, 1783) & F G & & & & & 2 & & 2 & 2 & & 15 & & 2 & 2 \\
\hline Cathartiformes & & & & & & & & & & & & & & \\
\hline Cathartidae & & & & & & & & & & & & & & \\
\hline Cathartes aura (Linnaeus, 1758) & $\mathrm{F}$ & & 6 & & 2 & 1 & & 1 & 1 & & 3 & & 1 & 2 \\
\hline Coragyps atratus (Bechstein, 1793) & $\mathrm{F}$ & & 12 & 3 & 6 & 15 & 4 & 2 & 8 & 4 & 8 & 2 & 230 & 12 \\
\hline Accipitriformes & & & & & & & & & & & & & & \\
\hline Accipitridae & & & & & & & & & & & & & & \\
\hline Leptodon cayanensis (Latham, 1790) & & & 2 & & & & 2 & 2 & & 1 & & & & \\
\hline Elanoides forficatus (Linnaeus, 1758) & & & 2 & & & & 2 & & & & & & & \\
\hline Gampsonyx swainsonii Vigors, 1825 & $\mathrm{~F}$ & & & & & & & & & & & & & 1 \\
\hline Elanus leucurus (Vieillot, 1818) & & & 1 & & & & & & & & & & 1 & 1 \\
\hline Accipiter striatus Vieillot, 1808 & & & & & & & & & & & 1 & & & \\
\hline Ictinia plumbea (Gmelin, 1788) & $\mathrm{F}$ & & 5 & 2 & & 3 & 3 & 1 & 1 & & & 1 & 4 & \\
\hline Rostrhamus sociabilis (Vieillot, 1817) & $\mathrm{F}$ & & & 1 & & & & & & & & & & \\
\hline Geranospiza caerulescens (Vieillot, 1817) & & & 1 & & & & & & & & 1 & & & \\
\hline Heterospizias meridionalis (Latham, 1790) & G & & & & & 2 & & 1 & & & & & & 1 \\
\hline Urubitinga coronata (Vieillot, 1817) & & AM & & & & & 1 & & & & & & & \\
\hline Rupornis magnirostris (Gmelin, 1788) & F G & & 7 & 3 & 2 & 4 & 2 & 32 & 2 & 2 & 2 & 4 & 2 & 2 \\
\hline
\end{tabular}

continuação - Tabela 2

continuation - Table 2

\begin{tabular}{|c|c|c|c|c|c|c|c|c|c|c|c|c|c|c|c|c|}
\hline Nome do Táxon & & & An & As & $\mathrm{Av}$ & $\mathrm{Ba}$ & $\mathrm{Bu}$ & $\mathrm{Br}$ & $\mathrm{Ca}$ & It & Ip & Ir & Ma & $\mathrm{Pa}$ & $\mathrm{RP}$ & SM \\
\hline Buteo brachyurus Vieillot, 1816 & G & & & 1 & & 1 & 1 & & & 1 & 1 & & 1 & & 1 & \\
\hline Spizaetus tyrannus (Wied, 1820) & G & AM & & & & & & & & 2 & & & & & & \\
\hline Gruiformes & & & & & & & & & & & & & & & & \\
\hline Aramidae & & & & & & & & & & & & & & & & \\
\hline Aramus guarauna (Linnaeus, 1766) & $\mathrm{F}$ & & & & & & & & & & & & 1 & & & \\
\hline Rallidae & & & & & & & & & & & & & & & & \\
\hline Aramides cajaneus (Statius Muller, 1776) & G & & 2 & & & 2 & 3 & & 1 & & & & & 2 & 5 & 2 \\
\hline Aramides saracura (Spix, 1825) & G & MT & 7 & & & & & & & & & & & & & \\
\hline Laterallus melanophaius (Vieillot, 1819) & & & 2 & & 2 & & & & & & & & 1 & & & \\
\hline Laterallus xenopterus Conover, 1934 & G & AM & & & & & & & 1 & & & & & & & 2 \\
\hline Mustelirallus albicollis (Vieillot, 1819) & G & & & 2 & & & & & 2 & & & & 1 & & 2 & 2 \\
\hline Pardirallus nigricans (Vieillot, 1819) & G & & & & & & 4 & & 1 & & 2 & & & 2 & & 2 \\
\hline Gallinula galeata (Lichtenstein, 1818) & F G & & & 3 & & & & & & & & & 2 & 4 & & \\
\hline Porphyrio martinicus (Linnaeus, 1766) & $\mathrm{F}$ & & & & & & & & & & & & 1 & & & \\
\hline Charadriiformes & & & & & & & & & & & & & & & & \\
\hline Charadriidae & & & & & & & & & & & & & & & & \\
\hline Vanellus chilensis (Molina, 1782) & F G & & 4 & 4 & 4 & 2 & 4 & & 1 & 4 & & 2 & 2 & 4 & 2 & 4 \\
\hline Scolopacidae & & & & & & & & & & & & & & & & \\
\hline Gallinago paraguaiae (Vieillot, 1816) & G & & & & & & & & & & & & 1 & & & \\
\hline Gallinago undulata (Boddaert, 1783) & G & & & & & & & & 1 & & & & & & & \\
\hline Jacanidae & & & & & & & & & & & & & & & & \\
\hline Jacana jacana (Linnaeus, 1766) & $\mathrm{F}$ & & 2 & 3 & 2 & & & & 1 & & & & 1 & 2 & & \\
\hline Columbiformes & & & & & & & & & & & & & & & & \\
\hline Columbidae & & & & & & & & & & & & & & & & \\
\hline Columbina talpacoti (Temminck, 1810) & F G & & 22 & & 6 & 2 & 10 & 1 & 1 & 6 & 4 & 8 & 5 & 8 & 8 & 6 \\
\hline Columbina squammata (Lesson, 1831) & F G & & 1 & 4 & 4 & 2 & 8 & & 1 & & & & 7 & & 2 & 6 \\
\hline Claravis pretiosa (Ferrari-Perez, 1886) & G & & 1 & & & & 2 & & 1 & & & & & & 2 & \\
\hline Columba livia Gmelin, 1789 & & & & & 12 & & & & & & & & & & & \\
\hline
\end{tabular}

AM 2

Patagioenas speciosa (Gmelin, 1789)

Patagioenas picazuro (Temminck, 1813)

Patagioenas cayennensis (Bonnaterre, 1792)

Zenaida auriculata (Des Murs, 1847)

$\begin{array}{lllllllllllll}33 & 10 & 26 & 4 & 10 & 6 & 10 & 8 & 20 & 9 & 20 & 10 & 23\end{array}$

Leptotila verreauxi Bonaparte, 1855

$\begin{array}{lllllllllllll}9 & 2 & 6 & 8 & 1 & 12 & 2 & 10 & 6 & 3 & 6\end{array}$

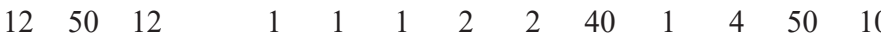

$\begin{array}{lllllllllllll}53 & 38 & 6 & 6 & 33 & 8 & 10 & 4 & 8 & 9 & 10 & 29 & 8\end{array}$

Leptotila rufaxilla (Richard \& Bernard, 1792)

Geotrygon montana (Linnaeus, 1758)

Cuculiformes

Cuculidae

Piaya cayana (Linnaeus, 1766)

$\begin{array}{llllllllllllll}\text { G } & 10 & 5 & 2 & 4 & 4 & 2 & 3 & 1 & 2 & 3 & 2 & 8 & 2\end{array}$ 
continuação - Tabela 2

continuation - Table 2

\begin{tabular}{|c|c|c|c|c|c|c|c|c|c|c|c|c|c|c|c|}
\hline Nome do Táxon & & & An & As & $\mathrm{Av}$ & $\mathrm{Ba}$ & $\mathrm{Bu} \mathrm{E}$ & $\mathrm{Br}$ & $\mathrm{Ca}$ & Ip & Ir & $\mathrm{Ma}$ & $\mathrm{Pa}$ & RP & SM \\
\hline Crotophaga ani Linnaeus, 1758 & G & & 12 & 8 & 6 & 8 & 8 & & 1 & 6 & & 5 & 6 & 12 & 6 \\
\hline Guira guira (Gmelin, 1788) & G & & 12 & 12 & 6 & & 8 & 1 & 1 & 6 & 6 & 3 & 8 & 8 & 8 \\
\hline Tapera naevia (Linnaeus, 1766) & G & & 6 & & 1 & & & & & 1 & 1 & 2 & 1 & 1 & 2 \\
\hline Dromососсух раvoninus Pelzeln, 1870 & & & & 1 & & & & & & & & & & & \\
\hline Strigiformes & & & & & & & & & & & & & & & \\
\hline Tytonidae & & & & & & & & & & & & & & & \\
\hline Tyto furcata (Temminck, 1827) & G & & 1 & & & & & & & & & 1 & 1 & & \\
\hline Strigidae & & & & & & & & & & & & & & & \\
\hline Megascops choliba (Vieillot, 1817) & G & & 2 & 2 & & & 2 & & 1 & & 2 & 1 & 1 & 1 & \\
\hline Pulsatrix perspicillata (Latham, 1790) & & & & & & & 4 & & & & & & & & \\
\hline Athene cunicularia (Molina, 1782) & F G & & 1 & 2 & & & 2 & & 1 & & & 1 & 2 & 1 & 4 \\
\hline Asio clamator (Vieillot, 1808) & & & 2 & & & & & & & & & & & & \\
\hline Asio stygius (Wagler, 1832) & G & & & & & & & & & & 2 & & & & \\
\hline Nyctibiiformes & & & & & & & & & & & & & & & \\
\hline Nyctibiidae & & & & & & & & & & & & & & & \\
\hline Nyctibius griseus (Gmelin, 1789) & G & & 3 & 1 & & & 1 & & 1 & & & 1 & & & \\
\hline Caprimulgiformes & & & & & & & & & & & & & & & \\
\hline Caprimulgidae & & & & & & & & & & & & & & & \\
\hline Antrostomus rufus (Boddaert, 1783) & G & & 4 & 6 & & & 3 & 2 & 1 & & & & & & \\
\hline Lurocalis semitorquatus (Gmelin, 1789) & G & & 2 & 4 & & 1 & 2 & & & & 2 & & 1 & & \\
\hline Nyctidromus albicollis (Gmelin, 1789) & G & & 11 & 10 & & & 4 & 1 & 1 & & 3 & 1 & 4 & 8 & \\
\hline Hydropsalis parvula (Gould, 1837) & G & & & 1 & & & & & & & 2 & & & & \\
\hline Hydropsalis longirostris (Bonaparte, 1825) & & & & & & & & & & & 1 & & & & \\
\hline Hydropsalis torquata (Gmelin, 1789) & & & & & & & & & & & & & 2 & & \\
\hline Hydropsalis forcipata (Nitzsch, 1840) & & MT & & & & & & & & & 1 & & & & \\
\hline Apodiformes & & & & & & & & & & & & & & & \\
\hline Apodidae & & & & & & & & & & & & & & & \\
\hline Streptoprocne zonaris (Shaw, 1796) & & & 4 & & & & & 2 & & & & & 4 & & \\
\hline Streptoprocne biscutata (Sclater, 1866) & G & & & & & & & & & 252 & 40 & & & & \\
\hline Chaetura meridionalis Hellmayr, 1907 & G & & & & & 10 & & & & & & 1 & & 1 & \\
\hline Trochilidae & & & & & & & & & & & & & & & \\
\hline Phaethornis pretrei (Lesson \& Delattre, 1839) & & & 8 & 5 & 1 & 6 & 4 & 3 & 2 & 1 & 1 & 4 & 1 & 1 & 2 \\
\hline Eupetomena macroura (Gmelin, 1788) & & & 5 & 1 & & 2 & & & 2 & & 2 & & 1 & & 1 \\
\hline Aphantochroa cirrochloris (Vieillot, 1818) & G & & & & & & & & & & & & & & \\
\hline Florisuga fusca (Vieillot, 1817) & $\mathrm{F}$ & MT & 7 & & & & & 1 & & & 1 & & 1 & & \\
\hline Anthracothorax nigricollis (Vieillot, 1817) & & & 2 & & & & & & & & & 2 & 1 & & \\
\hline Chlorostilbon lucidus (Shaw, 1812) & & & 6 & 5 & 1 & & 3 & 1 & 1 & 1 & 3 & 1 & 1 & 5 & 1 \\
\hline Thalurania furcata (Gmelin, 1788) & & AM & & & & 2 & & & & & & & & & \\
\hline
\end{tabular}

continuação - Tabela 2

continuation - Table 2

\begin{tabular}{|c|c|c|c|c|c|c|c|c|c|c|c|c|c|c|c|c|}
\hline Nome do Táxon & & & An & As & $\mathrm{Av}$ & $\mathrm{Ba}$ & $\mathrm{Bu}$ & $\mathrm{Br}$ & $\mathrm{Ca}$ & It & Ip & Ir & Ма & $\mathrm{Pa}$ & RP & SM \\
\hline Thalurania glaucopis (Gmelin, 1788) & F G & MT & 7 & & & 2 & & 3 & & & & & & 2 & & \\
\hline Hylocharis chrysura (Shaw, 1812) & G & & 7 & 11 & 4 & 2 & 6 & 1 & 2 & 1 & 2 & & 5 & & 2 & \\
\hline Leucochloris albicollis (Vieillot, 1818) & FG & MT & 3 & & & & & & & & & 6 & & & & \\
\hline Amazilia fimbriata (Gmelin, 1788) & & & & & & 1 & & & & & & & & & & \\
\hline Amazilia lactea (Lesson, 1832) & $\mathrm{F}$ & & 10 & & 2 & 3 & 2 & 1 & 1 & & 2 & 1 & & 1 & 1 & \\
\hline Heliomaster squamosus (Temminck, 1823) & $\mathrm{F}$ & & & & 1 & & & & 1 & & & & & & & \\
\hline Calliphlox amethystina (Boddaert, 1783) & & & 2 & & & & & & & & & & & & & \\
\hline Trogoniformes & & & & & & & & & & & & & & & & \\
\hline Trogonidae & & & & & & & & & & & & & & & & \\
\hline Trogon surrucura Vieillot, 1817 & F G & MT & 16 & 6 & & & & 3 & & 1 & 1 & 3 & & & & \\
\hline Coraciiformes & & & & & & & & & & & & & & & & \\
\hline Alcedinidae & & & & & & & & & & & & & & & & \\
\hline Megaceryle torquata (Linnaeus, 1766) & & & 1 & 1 & 1 & & & & & & & 1 & & 1 & 1 & \\
\hline Chloroceryle amazona (Latham, 1790) & $\mathrm{F}$ & & 2 & 1 & & & & & & & & & & & & \\
\hline Chloroceryle americana (Gmelin, 1788) & & & 1 & & & & & & & & & & & & & \\
\hline Momotidae & & & & & & & & & & & & & & & & \\
\hline Baryphthengus ruficapillus (Vieillot, 1818) & G & MT & & & & & 6 & 1 & & & & 2 & 1 & & & \\
\hline Galbuliformes & & & & & & & & & & & & & & & & \\
\hline Galbulidae & & & & & & & & & & & & & & & & \\
\hline Galbula ruficauda Cuvier, 1816 & F G & & & 1 & 2 & 4 & 2 & & 1 & & & & 1 & & 2 & 2 \\
\hline Bucconidae & & & & & & & & & & & & & & & & \\
\hline Nystalus chacuru (Vieillot, 1816) & G & & 1 & & & & & & 1 & & & & & & & \\
\hline Malacoptila striata (Spix, 1824) & $\mathrm{F}$ & MT & 11 & & & & & & & & & & & & & \\
\hline Piciformes & & & & & & & & & & & & & & & & \\
\hline Ramphastidae & & & & & & & & & & & & & & & & \\
\hline Ramphastos toco Statius Muller, 1776 & F G & & 7 & & 4 & 14 & 2 & 3 & 4 & & & & 1 & 6 & & 6 \\
\hline Ramphastos dicolorus Linnaeus, 1766 & F G & MT & & & & & & & & & & 2 & & & & \\
\hline Picidae & & & & & & & & & & & & & & & & \\
\hline Picumnus temminckii Lafresnaye, 1845 & G & MT & 7 & & & & & 3 & & 4 & 2 & 3 & & 2 & & \\
\hline Picumnus albosquamatus d'Orbigny, 1840 & F G & & & 2 & 2 & 6 & 10 & & 2 & & & & 4 & & 10 & 2 \\
\hline Melanerpes candidus (Otto, 1796) & F G & & 10 & 3 & 5 & 2 & 8 & & & & & & 1 & & 2 & 4 \\
\hline Veniliornis passerinus (Linnaeus, 1766) & G & & & 3 & 1 & 3 & 5 & & 1 & & & & 2 & & 4 & 2 \\
\hline Veniliornis spilogaster (Wagler, 1827) & G & MT & 5 & & & & & 3 & & 6 & 1 & 4 & & 2 & & \\
\hline Colaptes melanochloros (Gmelin, 1788) & G & & 2 & 1 & & 1 & & 2 & & 1 & 2 & 2 & 1 & 2 & 1 & 2 \\
\hline Colaptes campestris (Vieillot, 1818) & G & & 8 & 2 & 2 & 4 & 2 & 2 & 2 & & 2 & 4 & 3 & 2 & 1 & 2 \\
\hline Celeus flavescens (Gmelin, 1788) & G & & 6 & 5 & & & & & & 1 & 1 & & & 1 & & \\
\hline Dryocopus lineatus (Linnaeus, 1766) & G & & 5 & 1 & 1 & & 6 & 3 & & 2 & 1 & & 1 & 2 & 1 & \\
\hline ampephilus robustus (Lichtenstein, & G & MT & 5 & & 2 & & & & & & & & & & & \\
\hline
\end{tabular}


continuação - Tabela 2

continuation - Table 2

\begin{tabular}{|c|c|c|c|c|c|c|c|c|c|c|c|c|c|c|c|c|}
\hline Nome do Táxon & & & An & As & $\mathrm{Av}$ & $\mathrm{Ba}$ & $\mathrm{Bu}$ & $\mathrm{Br}$ & $\mathrm{Ca}$ & It & Ip & Ir & Мa & $\mathrm{Pa}$ & RP & SM \\
\hline Campephilus melanoleucos (Gmelin, 1788) & FG & & & & & & & & & & & & & & 3 & \\
\hline Cariamiformes & & & & & & & & & & & & & & & & \\
\hline Cariamidae & & & & & & & & & & & & & & & & \\
\hline Cariama cristata (Linnaeus, 1766) & F G & & 4 & 3 & 2 & 3 & 3 & & 2 & 2 & 2 & 2 & 1 & 2 & & 2 \\
\hline Falconiformes & & & & & & & & & & & & & & & & \\
\hline Falconidae & & & & & & & & & & & & & & & & \\
\hline Caracara plancus (Miller, 1777) & F G & & 2 & 2 & 2 & 4 & 2 & 1 & 1 & 3 & 1 & 2 & 1 & 2 & 4 & 2 \\
\hline Milvago chimachima (Vieillot, 1816) & FG & & 7 & 3 & 2 & 3 & 2 & 2 & 3 & & 2 & 2 & 1 & 2 & 1 & 2 \\
\hline Herpetotheres cachinnans (Linnaeus, 1758) & G & & 7 & & 2 & 3 & 2 & 1 & 1 & & & 2 & 2 & & 1 & 2 \\
\hline Micrastur semitorquatus (Vieillot, 1817) & G & & 3 & & & & & & & & & & & & & \\
\hline Falco sparverius Linnaeus, 1758 & $\mathrm{~F}$ & & & 1 & 2 & & 1 & & & & 1 & 1 & 1 & & & 2 \\
\hline Falco femoralis Temminck, 1822 & $\mathrm{~F}$ & & & 3 & & & & & 1 & & & & & & 3 & \\
\hline Psittaciformes & & & & & & & & & & & & & & & & \\
\hline Psittacidae & & & & & & & & & & & & & & & & \\
\hline Primolius maracana (Vieillot, 1816) & G & AM & & & & & & & & & & & 2 & & & \\
\hline Psittacara leucophthalmus (Statius Muller, 1776) & FG & & 16 & 14 & 16 & 20 & 26 & & 1 & & & & 3 & & 32 & 12 \\
\hline Aratinga auricapillus (Kuhl, 1820) & G & & & & & & & & & & & & & & 14 & \\
\hline Eupsittula aurea (Gmelin, 1788) & F G & & & & & 24 & & & 4 & & & & 1 & & & 10 \\
\hline Pyrrhura frontalis (Vieillot, 1817) & FG & MT & & & & & & & & & & 30 & & & & \\
\hline Forpus xanthopterygius (Spix, 1824) & $\mathrm{F}$ & & 18 & 4 & & 6 & 6 & 2 & & 2 & & & 4 & 4 & & 4 \\
\hline Brotogeris tirica (Gmelin, 1788) & $\mathrm{F}$ & MT & & & & & & & & & & & 5 & & & \\
\hline Brotogeris chiriri (Vieillot, 1818) & FG & & & 18 & & 10 & 22 & & 1 & & & 5 & & & 6 & 20 \\
\hline Pionus maximiliani (Kuhl, 1820) & FG & & & & & & 11 & 2 & & 4 & & & 1 & 6 & & \\
\hline Amazona amazonica (Linnaeus, 1766) & G & AM & & & & & & & & & & & & & 6 & \\
\hline Amazona aestiva (Linnaeus, 1758) & FG & & 2 & & & & 4 & & 3 & & & & & & & \\
\hline Passeriformes & & & & & & & & & & & & & & & & \\
\hline Thamnophilidae & & & & & & & & & & & & & & & & \\
\hline Formicivora rufa (Wied, 1831) & G & & 1 & 8 & & & & & & & & & & & & 2 \\
\hline Dysithamnus mentalis (Temminck, 1823) & G & & 18 & & 2 & & & 4 & & 10 & & 2 & & 4 & 2 & \\
\hline Herpsilochmus atricapillus Pelzeln, 1868 & G & & & & & & 14 & & & & & & 1 & & 35 & 6 \\
\hline Herpsilochmus longirostris Pelzeln, 1868 & G & $\begin{array}{l}\mathrm{AM} \\
\mathrm{CE}\end{array}$ & & & & 12 & & & & & & & & & & \\
\hline Thamnophilus doliatus (Linnaeus, 1764) & F G & & & 4 & 2 & 6 & 10 & & 2 & & & & 3 & & 15 & 4 \\
\hline Thamnophilus ruficapillus Vieillot, 1816 & G & & 4 & & & & & & & & 2 & 2 & & & & \\
\hline Thamnophilus torquatus Swainson, 1825 & & & & & & 1 & & & & & & & & & & \\
\hline Thamnophilus pelzelni Hellmayr, 1924 & G & & 8 & 23 & & 4 & 23 & & 2 & & & & & & 22 & 6 \\
\hline Thamnophilus caerulescens Vieillot, 1816 & G & & 37 & 2 & 4 & & 4 & 6 & & 6 & 4 & 6 & 4 & 4 & & \\
\hline Taraba major (Vieillot, 1816) & G & & & 2 & & & 5 & & & & & & 4 & & 3 & 2 \\
\hline
\end{tabular}

continuação - Tabela 2

continuation - Table 2

\begin{tabular}{|c|c|c|c|c|c|c|c|c|c|c|c|c|c|c|c|c|}
\hline \multicolumn{3}{|l|}{ Nome do Táxon } & \multirow[t]{2}{*}{ An } & \multirow[t]{2}{*}{ As } & \multirow[t]{2}{*}{$\mathrm{Av}$} & \multirow[t]{2}{*}{$\mathrm{Ba}$} & \multirow{2}{*}{$\frac{\mathrm{Bu}}{11}$} & \multirow[t]{2}{*}{$\mathrm{Br}$} & \multirow[t]{2}{*}{$\mathrm{Ca}$} & \multirow[t]{2}{*}{ It } & \multirow[t]{2}{*}{ Ip } & \multirow[t]{2}{*}{ Ir } & \multirow[t]{2}{*}{ Ma } & \multirow[t]{2}{*}{$\mathrm{Pa}$} & \multirow[t]{2}{*}{$\mathrm{RP}$} & \multirow[t]{2}{*}{ SM } \\
\hline Hypoedaleus guttatus (Vieillot, 1816) & G & MT & & & & & & & & & & & & & & \\
\hline Batara cinerea (Vieillot, 1819) & G & & & & & & & & & & & 2 & & & & \\
\hline Mackenziaena leachii (Such, 1825) & G & MT & 2 & & & & & & & & & 1 & & & & \\
\hline Mackenziaena severa (Lichtenstein, 1823) & G & MT & 8 & & & & & 2 & & & & & & 2 & & \\
\hline Pyriglena leucoptera (Vieillot, 1818) & G & MT & & & & & & 6 & & 8 & 6 & 6 & & 2 & & \\
\hline Drymophila ferruginea (Temminck, 1822) & G & MT & 1 & & & & & & & 1 & & & & & & \\
\hline Drymophila rubricollis (Bertoni, 1901) & & MT & & & & & & & & & & 1 & & & & \\
\hline Drymophila malura (Temminck, 1825) & G & MT & 8 & & 2 & & & 2 & & & 2 & 1 & & 4 & & \\
\hline \multicolumn{17}{|l|}{ Conopophagidae } \\
\hline Conopophaga lineata (Wied, 1831) & FG & & 16 & 6 & 2 & & 4 & 2 & & 4 & 2 & 2 & & 2 & 4 & \\
\hline \multicolumn{17}{|l|}{ Rhinocryptidae } \\
\hline Eleoscytalopus indigoticus (Wied, 1831) & G & MT & 14 & & & & & & & & & & & & & \\
\hline Psilorhamphus guttatus (Ménétriès, 1835) & G & MT & & & & & & 2 & & & 1 & & & & & \\
\hline \multicolumn{17}{|l|}{ Formicariidae } \\
\hline Chamaeza campanisona (Lichtenstein, 1823) & G & & & & & & & & & 6 & & & & & & \\
\hline \multicolumn{17}{|l|}{ Scleruridae } \\
\hline Sclerurus scansor (Ménétriès, 1835) & G & MT & 1 & & & & & & & & & & & & & \\
\hline \multicolumn{17}{|l|}{ Dendrocolaptidae } \\
\hline Sittasomus griseicapillus (Vieillot, 1818) & G & & 12 & & & & 15 & 6 & & 4 & & 1 & & 6 & & \\
\hline Xiphorhynchus fuscus (Vieillot, 1818) & G & MT & 8 & & & & & 3 & & 4 & & 1 & & 2 & & \\
\hline Lepidocolaptes angustirostris (Vieillot, 1818) & F G & & & 1 & 3 & 10 & & & 3 & & & & 4 & 1 & 3 & 4 \\
\hline Lepidocolaptes falcinellus(Cabanis\& Heine, 1859) & & MT & & & & & & & & & & 1 & & & & \\
\hline Campylorhamphus falcularius (Vieillot, 1822) & G & MT & & & & & & & & 1 & & & & & & \\
\hline Dendrocolaptes platyrostris Spix, 1825 & G & & 2 & & & & 2 & & & 4 & & 3 & & & & \\
\hline \multicolumn{17}{|l|}{ Xenopidae } \\
\hline Xenops rutilans Temminck, 1821 & & & 1 & & & & 2 & & & 3 & & & & & & \\
\hline Furnariidae & & & & & & & & & & & & & & & & \\
\hline Furnarius rufus (Gmelin, 1788) & F G & & 4 & 2 & 2 & 2 & 2 & & & 2 & & 4 & 4 & 2 & 2 & 2 \\
\hline Lochmias nematura (Lichtenstein, 1823) & G & & & & & & & & & & & 2 & & 1 & & \\
\hline Automolus leucophthalmus (Wied, 1821) & G & MT & 15 & 5 & & 2 & 11 & 4 & & 12 & 1 & & & 2 & & \\
\hline Syndactyla rufosuperciliata (Lafresnaye, 1832) & G & & & & & & & & & & 1 & 4 & & & & \\
\hline Leptasthenura setaria (Temminck, 1824) & G & MT & & & & & & & & 1 & & 2 & & & & \\
\hline Phacellodomus ferrugineigula (Pelzeln, 1858) & & MT & & & 2 & & & & & & 1 & & & 2 & & \\
\hline Anumbius аппитbi (Vieillot, 1817) & & & & & & & & & & & & & & & & \\
\hline Certhiaxis cinnamomeus (Gmelin, 1788) & G & & 1 & & & 2 & & & 1 & & & & 1 & 2 & & 2 \\
\hline Synallaxis ruficapilla Vieillot, 1819 & G & MT & 21 & & 4 & & & 4 & & 6 & 2 & 5 & & 4 & & \\
\hline Synallaxis frontalis Pelzeln, 1859 & G & & & 15 & & 12 & 8 & & 1 & & & & 4 & & 18 & \\
\hline vnallaxis albescens Temminck, 182 & G & & & & & & & & & & & & & & & \\
\hline
\end{tabular}


continuação - Tabela 2

continuation - Table 2

\begin{tabular}{|c|c|c|c|c|c|c|c|c|c|c|c|c|c|c|}
\hline Nome do Táxon & & & An $A$ & As & $\mathrm{Av} \mathrm{Ba}$ & $\mathrm{Ba} \mathrm{Bu}$ & $\mathrm{Br}$ & $\mathrm{Ca}$ It & Ip & Ir & Ma & $\mathrm{Pa} \mathrm{I}$ & RP & SM \\
\hline Synallaxis spixi Sclater, 1856 & G & & 5 & & 3 & 5 & 3 & 2 & 1 & 4 & & 2 & & 2 \\
\hline Cranioleuca vulpina (Pelzeln, 1856) & G & & & & & 1 & & & & & & & & 2 \\
\hline Cranioleuca pallida (Wied, 1831) & G & MT & & & & & 1 & 6 & 2 & 6 & & & & \\
\hline \multicolumn{15}{|l|}{ Pipridae } \\
\hline Manacus manacus (Linnaeus, 1766) & $\mathrm{F}$ & & & & & & & 2 & & & & & & \\
\hline Chiroxiphia caudata (Shaw \& Nodder, 1793) & G & MT & 49 & & & & 12 & 14 & 3 & 6 & & 10 & & \\
\hline Antilophia galeata (Lichtenstein, 1823) & FG & $\mathrm{CE}$ & & 22 & & 20 & & 5 & & & & & & 6 \\
\hline \multicolumn{15}{|l|}{ Tityridae } \\
\hline Schiffornis virescens (Lafresnaye, 1838) & G & MT & 14 & & & & 8 & 6 & 1 & 3 & & 4 & & \\
\hline Tityra inquisitor (Lichtenstein, 1823) & & & & & & 1 & & & & & & & & \\
\hline Tityra cayana (Linnaeus, 1766) & & & & & & & & & & & 1 & 1 & & \\
\hline Pachyramphus castaneus (Jardine \& Selby, 1827) & G & & & & & & & 1 & 1 & & & & & \\
\hline Pachyramphus polychopterus (Vieillot, 1818) & G & & 12 & & 1 & 1 & 1 & 2 & & & & & & \\
\hline Pachyramphus validus (Lichtenstein, 1823) & G & & 4 & 2 & & & 1 & 2 & 2 & 2 & 1 & 2 & & \\
\hline \multicolumn{15}{|l|}{ Cotingidae } \\
\hline Carpornis cucullata (Swainson, 1821) & G & MT & & & & & & & & 3 & & & & \\
\hline Pyroderus scutatus (Shaw, 1792) & $\mathrm{F}$ & $\begin{array}{l}\text { AM } \\
\text { MT }\end{array}$ & 4 & & & & 1 & & & & & & & \\
\hline Procnias nudicollis (Vieillot, 1817) & G & $\begin{array}{l}\text { AM } \\
\text { MT }\end{array}$ & 13 & & & & 8 & & & 6 & & 2 & & \\
\hline \multicolumn{15}{|l|}{ Platyrinchidae } \\
\hline Platyrinchus mystaceus Vieillot, 1818 & F G & & 14 & 2 & 1 & 2 & 3 & 5 & 2 & 2 & & 4 & & \\
\hline \multicolumn{15}{|l|}{ Rhynchocyclidae } \\
\hline Leptopogon amaurocephalus Tschudi, 1846 & G & & 3 & 4 & 1 & 10 & 1 & 2 & 2 & & 1 & 2 & 3 & 2 \\
\hline Corythopis delalandi (Lesson, 1830) & F G & & 38 & & & 6 & 10 & 5 & & & & 3 & & \\
\hline Phylloscartes ventralis (Temminck, 1824) & G & & & & & & & 2 & 2 & 2 & & & & \\
\hline Tolmomyias sulphurescens (Spix, 1825) & G & & 15 & & 2 & 3 & 2 & 8 & 2 & 3 & & 4 & & \\
\hline Todirostrum poliocephalum (Wied, 1831) & G & MT & 7 & & 1 & & 3 & 10 & 1 & & & 3 & & \\
\hline Todirostrum cinereum (Linnaeus, 1766) & G & & 3 & 2 & 2 & 6 & & 1 & & & 2 & 2 & 9 & 4 \\
\hline Poecilotriccus plumbeiceps (Lafresnaye, 1846) & G & & 1 & & 1 & & & 2 & 1 & 4 & & & & \\
\hline Poecilotriccus latirostris (Pelzeln, 1868) & G & & & & & & & & & & 4 & & & \\
\hline Myiornis auricularis (Vieillot, 1818) & G & MT & 12 & & 2 & 8 & 4 & & & & 1 & & 2 & \\
\hline Hemitriccus diops (Temminck, 1822) & $\mathrm{F}$ & MT & & & & & & 5 & 5 & & & & & \\
\hline Hemitriccus orbitatus (Wied, 1831) & G & MT & 11 & & & 2 & 6 & & & & & 4 & & \\
\hline Hemitriccus nidipendulus (Wied, 1831) & G & MT & 2 & & & & & & 1 & 1 & & 1 & & \\
\hline $\begin{array}{l}\text { Hemitriccus margaritaceiventer (d‘Orbigny } \\
\& \text { Lafresnaye, 1837) }\end{array}$ & G & & & 6 & 4 & 4 & & & & & & & & \\
\hline \multicolumn{15}{|l|}{ Tyrannidae } \\
\hline Hirundinea ferruginea (Gmelin, 1788) & $\mathrm{F}$ & & & & & & & & & 1 & 1 & & & \\
\hline
\end{tabular}

continuação - Tabela 2

continuation - Table 2

\begin{tabular}{|c|c|c|c|c|c|c|c|c|c|c|c|c|c|c|c|c|}
\hline Nome do Táxon & & & An & As & $\mathrm{Av}$ & $\mathrm{Ba}$ & $\mathrm{Bu}$ & $\mathrm{Br}$ & $\mathrm{Ca}$ & It & Ip & Ir & Ma & $\mathrm{Pa}$ & RP & SM \\
\hline Euscarthmus meloryphus Wied, 1831 & $\mathrm{~F}$ & & & 12 & & & 5 & & & & & & & & & \\
\hline Camptostoma obsoletum (Temminck, 1824) & G & & 9 & 10 & 2 & 2 & 3 & 3 & 4 & 6 & 2 & 2 & 5 & 4 & 1 & 2 \\
\hline Elaenia flavogaster (Thunberg, 1822) & G & & 10 & 6 & 2 & 3 & 2 & 4 & 2 & 2 & 4 & 6 & 2 & 2 & 2 & \\
\hline Elaenia parvirostris Pelzeln, 1868 & G & & & & 2 & & & & & & & 6 & & & 2 & \\
\hline Elaenia mesoleuca (Deppe, 1830) & G & & & & & & 1 & 6 & & & & 12 & & & & \\
\hline Elaenia cristata Pelzeln, 1868 & G & AM & & & & 3 & & & & & & & & & & \\
\hline Elaenia chiriquensis Lawrence, 1865 & G & & & 2 & & 8 & & & 7 & & 2 & 4 & & & & \\
\hline Elaenia obscura (d'Orbigny \& Lafresnaye, 1837) & G & & 15 & 15 & 3 & 2 & 2 & & 1 & 1 & 4 & & & 2 & & \\
\hline Myiopagis caniceps (Swainson, 1835) & G & & & & & & 2 & & & & & & & & & \\
\hline Myiopagis viridicata (Vieillot, 1817) & & & & & & & 1 & & & & & & & & & \\
\hline Capsiempis flaveola (Lichtenstein, 1823) & G & & & 2 & & & & 2 & & & & & & & 3 & \\
\hline Phaeomyias murina (Spix, 1825) & G & & 4 & 15 & & & 2 & & 2 & & 2 & & & 2 & 3 & \\
\hline Phyllomyias fasciatus (Thunberg, 1822) & G & & & & & & 1 & & & & & & & & & \\
\hline Serpophaga subcristata (Vieillot, 1817) & G & & 2 & 1 & 1 & 3 & 2 & & 2 & & & 3 & & 1 & 1 & 2 \\
\hline Legatus leucophaius (Vieillot, 1818) & G & & & & & & & 2 & & & & & & & & \\
\hline Myiarchus swainsoni Cabanis \& Heine, 1859 & G & & 10 & 2 & & & 10 & 6 & 1 & 4 & 2 & 6 & & 6 & & \\
\hline Myiarchus ferox (Gmelin, 1789) & G & & 5 & 3 & 2 & 4 & 4 & 2 & 2 & 1 & 4 & 1 & 5 & 2 & 14 & 4 \\
\hline Myiarchus tyrannulus (Statius Muller, 1776) & G & & 3 & 2 & & 10 & 3 & & 3 & & 1 & & 1 & 2 & 2 & 8 \\
\hline Casiornis rufus (Vieillot, 1816) & & & 1 & 2 & & & & & & & & & & & & \\
\hline Pitangus sulphuratus (Linnaeus, 1766) & F G & & 8 & 2 & 4 & 5 & 2 & 4 & 2 & 4 & 3 & 6 & 4 & 4 & 8 & 6 \\
\hline Machetornis rixosa (Vieillot, 1819) & F G & & 4 & 2 & 2 & & & 2 & & & & 2 & 1 & 2 & & 2 \\
\hline Myiodynastes maculatus (Statius Muller, 1776) & G & & 13 & 8 & & 8 & 13 & 3 & 3 & 6 & 2 & 1 & 5 & 4 & 5 & \\
\hline Megarynchus pitangua (Linnaeus, 1766) & G & & 7 & 6 & 2 & 6 & 2 & 2 & 4 & 1 & & 1 & 4 & 3 & 4 & 4 \\
\hline Myiozetetes similis (Spix, 1825) & G & & 4 & 2 & 2 & 4 & 2 & 1 & 1 & & & & 1 & 2 & & 3 \\
\hline Tyrannus melancholicus Vieillot, 1819 & F G & & 10 & 6 & 4 & 8 & 2 & 4 & 6 & 6 & 8 & 8 & 4 & 4 & 4 & 4 \\
\hline Tyrannus savana Daudin, 1802 & F G & & 6 & 2 & 6 & 1 & & 2 & 1 & 5 & 3 & 4 & 1 & 2 & & \\
\hline Empidonomus varius (Vieillot, 1818) & G & & 4 & 8 & & 2 & & 1 & 2 & 2 & 3 & & 3 & 2 & & \\
\hline Colonia colonus (Vieillot, 1818) & F G & & 3 & & 2 & 1 & 3 & & 1 & 3 & & & 1 & 2 & 6 & 2 \\
\hline Myiophobus fasciatus (Statius Muller, 1776) & G & & 3 & 4 & 1 & 2 & 2 & 2 & 2 & & 2 & 3 & & 1 & 1 & 2 \\
\hline Pyrocephalus rubinus (Boddaert, 1783) & $\mathrm{F}$ & & & & & & & & & & & & & & & 1 \\
\hline Fluvicola nengeta (Linnaeus, 1766) & $\mathrm{F}$ & & & & & 3 & & & & & & & & & & 2 \\
\hline Arundinicola leucocephala (Linnaeus, 1764) & $\mathrm{F}$ & & 3 & & & & & & 1 & & & & & & & 2 \\
\hline Gubernetes yetapa (Vieillot, 1818) & FG & $\mathrm{CE}$ & & & & & 1 & & 2 & & & & 2 & 2 & & 4 \\
\hline Cnemotriccus fuscatus (Wied, 1831) & G & & & 12 & & & 12 & & 1 & & & & 1 & & 2 & \\
\hline Lathrotriccus euleri (Cabanis, 1868) & G & & 15 & 10 & 2 & 2 & 12 & 8 & & 6 & 1 & & & 4 & 1 & \\
\hline Knipolegus nigerrimus (Vieillot, 1818) & $\mathrm{F}$ & MT & & & & & & & & & & 6 & & & & \\
\hline
\end{tabular}


continuação - Tabela 2

continuation - Table 2

\begin{tabular}{|c|c|c|c|c|c|c|c|c|c|c|c|c|c|c|c|}
\hline Nome do Táxon & & & An & As & Av $\mathrm{E}$ & Ba $\mathrm{B}$ & $\mathrm{Bu} \mathrm{F}$ & $\mathrm{Br} \mathrm{C}$ & $\mathrm{Ca}$ It & Ip & Ir & Мa & $\mathrm{Pa}$ & RP & SM \\
\hline Xolmis cinereus (Vieillot, 1816 ) & F G & & & & 2 & & 1 & 1 & & 1 & 2 & & 2 & & 2 \\
\hline Xolmis velatus (Lichtenstein, 1823) & & & 1 & & 2 & & 3 & & 2 & & & 1 & 2 & & 2 \\
\hline Muscipipra vetula (Lichtenstein, 1823) & G & MT & & & & & & & & & 2 & & & & \\
\hline \multicolumn{16}{|l|}{ Vireonidae } \\
\hline Cyclarhis gujanensis (Gmelin, 1789) & G & & 17 & 4 & 4 & 6 & 11 & 3 & 10 & 4 & 8 & 7 & 6 & 5 & 6 \\
\hline Hylophilus amaurocephalus (Nordmann, 1835) & & & & 2 & 2 & & 2 & & & 2 & & & & & \\
\hline Hylophilus poicilotis Temminck, 1822 & G & MT & & & & & & & & & 2 & & & & \\
\hline Vireo chivi (Vieillot, 1817) & G & & 11 & 37 & 3 & 2 & 231 & 10 & 12 & 4 & 10 & 1 & 10 & 1 & \\
\hline \multicolumn{16}{|l|}{ Corvidae } \\
\hline Cyanocorax cristatellus (Temminck, 1823) & F G & $\mathrm{CE}$ & 8 & 2 & 10 & 4 & 5 & & 2 & & & 1 & & & 12 \\
\hline Cyanocorax chrysops (Vieillot, 1818) & F G & & 22 & 20 & 6 & & 101 & 10 & 1 & 3 & & 3 & 20 & & 8 \\
\hline \multicolumn{16}{|l|}{ Hirundinidae } \\
\hline Pygochelidon cyanoleuca (Vieillot, 1817) & F G & & 16 & 6 & 12 & 4 & 2 & 4 & 10 & 0 & 12 & 2 & & 6 & 12 \\
\hline Stelgidopteryx ruficollis (Vieillot, 1817) & F G & & 14 & 6 & 4 & 2 & 10 & 6 & 1 & & 6 & 1 & 10 & 1 & 8 \\
\hline Progne tapera (Vieillot, 1817) & & & & & 2 & & & & & & & 1 & & & \\
\hline Progne chalybea (Gmelin, 1789) & F G & & 7 & 3 & & & & 4 & & & 10 & & 12 & & \\
\hline Tachycineta albiventer (Boddaert, 1783) & & & & 2 & & & & & & & & & 2 & & \\
\hline Tachycineta leucorrhoa (Vieillot, 1817) & $\mathrm{F}$ & & & & & & 6 & 2 & 1 & & 4 & & & & 4 \\
\hline Hirundo rustica Linnaeus, 1758 & & & & & & & 4 & & & & & & & & \\
\hline \multicolumn{16}{|l|}{ Troglodytidae } \\
\hline Troglodytes musculus Naumann, 1823 & G & & 3 & 3 & 3 & 10 & 5 & 2 & 2 & 2 & 4 & 5 & 4 & 3 & 6 \\
\hline Cantorchilus leucotis (Lafresnaye, 1845) & G & & & & & 12 & & & 4 & & & & & 27 & 8 \\
\hline \multicolumn{16}{|l|}{ Donacobiidae } \\
\hline Donacobius atricapilla (Linnaeus, 1766) & & & & & & & & & 2 & 2 & & & 4 & & \\
\hline \multicolumn{16}{|l|}{ Polioptilidae } \\
\hline Polioptila dumicola (Vieillot, 1817) & & & & & & & & & & & & & & 2 & \\
\hline \multicolumn{16}{|l|}{ Turdidae } \\
\hline Turdus flavipes Vieillot, 1818 & G & & & & & & & 1 & & & 1 & & & & \\
\hline Turdus leucomelas Vieillot, 1818 & FG & & 12 & 34 & 102 & 201 & 14 & 1 & 6 & 1 & 1 & 3 & 4 & 3 & 10 \\
\hline Turdus rufiventris Vieillot, 1818 & FG & & 11 & & 2 & & & 2 & 8 & 2 & 14 & & 6 & & \\
\hline Turdus amaurochalinus Cabanis, 1850 & FG & & 10 & 6 & 4 & & & 2 & 16 & 2 & 8 & & 2 & & \\
\hline Turdus subalaris (Seebohm, 1887) & & MT & 4 & 12 & & & & & 2 & 2 & 3 & & & & \\
\hline Turdus albicollis Vieillot, 1818 & F G & & 10 & & & & & 12 & 6 & 5 & 6 & & 8 & & \\
\hline \multicolumn{16}{|l|}{ Mimidae } \\
\hline Mimus saturninus (Lichtenstein, 1823) & F G & & 10 & 6 & 4 & 8 & 8 & & 1 & & 4 & 3 & 5 & 4 & 6 \\
\hline \multicolumn{16}{|l|}{ Motacillidae } \\
\hline Anthus lutescens Pucheran, 1855 & & & & & & & & & 1 & & & & 2 & & 2 \\
\hline
\end{tabular}

continuação - Tabela 2

continuation - Table 2

\begin{tabular}{|c|c|c|c|c|c|c|c|c|c|c|c|c|c|c|c|c|}
\hline \multicolumn{3}{|l|}{ Nome do Táxon } & An & As & $\mathrm{Av}$ & $\mathrm{Ba}$ & $\mathrm{Bu}$ & $\mathrm{Br}$ & $\mathrm{Ca}$ & It & Ip & Ir & Мa & $\mathrm{Pa}$ & RP & SM \\
\hline \multicolumn{17}{|l|}{ Passerellidae } \\
\hline Zonotrichia capensis (Statius Muller, 1776) & F G & & 12 & 2 & 10 & 16 & 2 & 4 & 4 & 8 & 16 & 25 & 5 & 10 & 3 & 8 \\
\hline Ammodramus humeralis (Bosc, 1792) & F G & & 2 & 1 & & 4 & & & 3 & 1 & & 2 & 3 & & & 12 \\
\hline Arremon flavirostris Swainson, 1838 & F G & & & 8 & & 2 & 4 & & & & & & 1 & & 3 & \\
\hline \multicolumn{17}{|l|}{ Parulidae } \\
\hline Setophaga pitiayumi (Vieillot, 1817) & G & & 15 & 6 & 4 & 6 & & 1 & 2 & 8 & 3 & 3 & & 4 & & \\
\hline Geothlypis aequinoctialis (Gmelin, 1789) & G & & 4 & 1 & 2 & & & 1 & 2 & 2 & 1 & 2 & & 2 & & \\
\hline Basileuterus culicivorus (Deppe, 1830) & F G & & 63 & 31 & 6 & 8 & 36 & 12 & 1 & 20 & 4 & 14 & & 18 & & 8 \\
\hline Myiothlypis flaveola Baird, 1865 & F G & & 6 & 21 & 6 & 12 & 25 & & 1 & & & & 7 & & 2 & 10 \\
\hline Myiothlypis leucoblephara (Vieillot, 1817) & G & MT & 21 & & 2 & & & 6 & & 14 & 2 & 12 & & 10 & & \\
\hline Myiothlypis leucophrys (Pelzeln, 1868) & $\mathrm{F}$ & $\begin{array}{l}\mathrm{AM} \\
\mathrm{CE}\end{array}$ & & & & & & & & & & & & & & 4 \\
\hline \multicolumn{17}{|l|}{ Icteridae } \\
\hline Psarocolius decumanus (Pallas, 1769) & F G & & 11 & & 4 & & & 6 & & & 1 & & & 6 & & \\
\hline Icterus pyrrhopterus (Vieillot, 1819) & G & & 1 & 1 & & 5 & & & 1 & & & 1 & 1 & & 3 & 2 \\
\hline Gnorimopsar chopi (Vieillot, 1819) & & & & & 6 & & & & 1 & & & 4 & & & & 4 \\
\hline Chrysomus ruficapillus (Vieillot, 1819) & G & & 8 & & & & & & & & & & 2 & & & \\
\hline Pseudoleistes guirahuro (Vieillot, 1819) & F G & & 6 & & 6 & & 8 & & 1 & & & & 2 & 8 & & 12 \\
\hline Molothrus oryzivorus (Gmelin, 1788) & $\mathrm{F}$ & & 3 & & & & & & & & & & & & & \\
\hline Molothrus bonariensis (Gmelin, 1789) & $\mathrm{F}$ & & 1 & 3 & 8 & 3 & & & 1 & 4 & 5 & 3 & 1 & 6 & & \\
\hline Sturnella superciliaris (Bonaparte, 1850) & F G & & & & & 1 & & & & & & & 1 & & & 6 \\
\hline \multicolumn{17}{|l|}{ Thraupidae } \\
\hline Pipraeidea melanonota (Vieillot, 1819) & F G & & & & & & & & & 2 & 1 & & & 1 & & \\
\hline Stephanophorus diadematus (Temminck, 1823) & F G & & & & & & & & & & & 8 & & & & \\
\hline Schistochlamys melanopis (Latham, 1790) & G & $\mathrm{AM}$ & & & & 1 & & & 1 & & & & & & & \\
\hline Schistochlamys ruficapillus (Vieillot, 1817) & F G & & & & 2 & & & & & & & 2 & & & & \\
\hline Tangara desmaresti (Vieillot, 1819) & G & MT & & & & & & & & & & 22 & & & & \\
\hline Tangara sayaca (Linnaeus, 1766) & G & & 14 & 8 & 4 & 6 & 14 & 8 & 5 & 8 & 8 & 12 & 8 & 4 & 6 & 8 \\
\hline Tangara palmarum (Wied, 1821) & G & & & & & 2 & & & & & & & & & & \\
\hline Tangara cayana (Linnaeus, 1766) & G & & 16 & 26 & 4 & 14 & 10 & 4 & 3 & 10 & 8 & 6 & 2 & 8 & & 12 \\
\hline Nemosia pileata (Boddaert, 1783) & G & & & 3 & & 6 & 5 & & 1 & & & & & & 9 & 4 \\
\hline Conirostrum speciosum (Temminck, 1824) & G & & 6 & 6 & & 6 & 12 & & 1 & 2 & & & 1 & & 31 & 6 \\
\hline Sicalis flaveola (Linnaeus, 1766) & F G & & 8 & & 10 & & & 4 & 1 & & & 40 & 1 & 10 & & 10 \\
\hline Sicalis luteola (Sparrman, 1789) & F G & & & & & 1 & & & & & & & & & & 4 \\
\hline Haplospiza unicolor Cabanis, 1851 & G & MT & & & & & & & & & & 2 & & & & \\
\hline Hemithraupis guira (Linnaeus, 1766) & G & & & 12 & & 2 & 16 & & 1 ? & 6 & & & & & 15 & \\
\hline Hemithraupis ruficapilla (Vieillot, 181 & G & MT & 1 & & & & & & & & & & & & & \\
\hline
\end{tabular}


continuação - Tabela 2

continuation - Table 2

\begin{tabular}{|c|c|c|c|c|c|c|c|c|c|c|c|c|c|c|c|c|}
\hline Nome do Táxon & & & An & As & $\mathrm{Av}$ & Ва 1 & $\mathrm{Bu}$ & $\mathrm{Br}$ & $\mathrm{Ca}$ & It & Ip & Ir 1 & Ма & $\mathrm{Pa} \quad \mathrm{R}$ & RP s & SM \\
\hline Volatinia jacarina (Linnaeus, 1766) & G & & 15 & 1 & 20 & 14 & & 1 & 1 & 4 & & 16 & 5 & 10 & 7 & 23 \\
\hline Eucometis penicillata (Spix, 1825) & G & AM & & & & 10 & & & & & & & & & 5 & \\
\hline Trichothraupis melanops (Vieillot, 1818) & G & & 11 & & & & & 6 & & 8 & 2 & & & 4 & & \\
\hline Coryphospingus cucullatus (Statius Muller, 1776) & F G & & & 2 & 8 & 8 & 6 & & 1 & & & & 4 & 6 & & 10 \\
\hline Tachyphonus coronatus (Vieillot, 1822) & F G & MT & 14 & & 2 & 1 & & 4 & & 5 & 2 & 2 & & 3 & 2 & \\
\hline Ramphocelus carbo (Pallas, 1764) & G & & 5 & 1 & 6 & 3 & 5 & & 1 & & & & 2 & & & 6 \\
\hline Tersina viridis (Illiger, 1811) & F G & & 15 & 6 & 8 & 6 & & 10 & 1 & 6 & 5 & 20 & 1 & 4 & 1 & 8 \\
\hline Dacnis cayana (Linnaeus, 1766) & F G & & 15 & 8 & & 4 & 4 & 1 & 1 & 2 & & 1 & & & 2 & 4 \\
\hline Coereba flaveola (Linnaeus, 1758) & F G & & 9 & & 2 & 8 & 8 & 2 & 1 & 6 & & & & 4 & 17 & \\
\hline Tiaris fuliginosus (Wied, 1830) & G & & & 1 & & 2 & & & & & & & & & 2 & \\
\hline Sporophila lineola (Linnaeus, 1758) & G & & 4 & & 4 & 2 & & & & & & & 5 & 2 & 2 & 4 \\
\hline Sporophila ardesiaca (Dubois, 1894) & & & & & & & & & & & & & 1 & & & \\
\hline Sporophila caerulescens (Vieillot, 1823) & G & & 10 & & 9 & 4 & 1 & 1 & 1 & & & 50 & 1 & 4 & 4 & 4 \\
\hline Sporophila angolensis (Linnaeus, 1766) & & AM & & & & & & & & & 1 & & & & & \\
\hline Embernagra platensis (Gmelin, 1789) & F G & AM & & & & & & & & & & 8 & & & & \\
\hline Emberizoides herbicola (Vieillot, 1817) & G & & & & & & & & 3 & & & & 1 & & & 5 \\
\hline Saltatricula atricollis (Vieillot, 1817) & FG & $\begin{array}{l}\mathrm{AM} \\
\mathrm{CE}\end{array}$ & & & & & & & 3 & & & & & & & 4 \\
\hline Saltator similis d‘Orbigny \& Lafresnaye, 1837 & F G & & 3 & 3 & & & 2 & 3 & 1 & 18 & 4 & 10 & & 4 & 2 & \\
\hline Saltator fuliginosus (Daudin, 1800) & G & MT & 14 & & & & & 1 & & & & & & & & \\
\hline Microspingus cabanisi Bonaparte, 1850 & G & & & & & & & & & & & 8 & & & & \\
\hline Thlypopsis sordida (d'Orbigny \& Lafiesnaye, 1837) & G & & 2 & & & 2 & 3 & & & 6 & & & & 2 & 15 & \\
\hline Cardinalidae & & & & & & & & & & & & & & & & \\
\hline Piranga flava (Vieillot, 1822) & $\mathrm{F}$ & & & & & 1 & & & 1 & & & & & & & \\
\hline Habia rubica (Vieillot, 1817) & G & & 28 & & 4 & & & 8 & & 8 & & & & 4 & & \\
\hline Amaurospiza moesta (Hartlaub, 1853) & & $\begin{array}{l}\text { AM } \\
\text { MT }\end{array}$ & & & & & & & & 1 & & & & & & \\
\hline $\begin{array}{l}\text { Cyanoloxia glaucocaerulea (d'Orbigny \& } \\
\text { Lafresnaye, 1837) }\end{array}$ & $\mathrm{F}$ & & & & & & & & & & & 1 & & & & \\
\hline Cyanoloxia brissonii (Lichtenstein, 1823) & G & AM & 1 & & & & & & & & & & & 1 & & \\
\hline Fringillidae & & & & & & & & & & & & & & & & \\
\hline Spinus magellanicus (Vieillot, 1805) & F G & & 8 & & 12 & & & 5 & & 6 & & 14 & & 10 & & \\
\hline Euphonia chlorotica (Linnaeus, 1766) & G & & 7 & 8 & 2 & 20 & 16 & 2 & 3 & 5 & 2 & & 4 & 4 & 5 & 4 \\
\hline Euphonia violacea (Linnaeus, 1758) & G & & 2 & & & & & & 1 & & & & & & 1 & \\
\hline Estrildidae & & & & & & & & & & & & & & & & \\
\hline Estrilda astrild (Linnaeus, 1758) & & & & & & & & & & & & & & & 8 & \\
\hline Passeridae & & & & & & & & & & & & & & & & \\
\hline Passer domesticus (Linnaeus, 1758) & $\mathrm{F}$ & & & 3 & 8 & & & & & 6 & & & 1 & 6 & & 6 \\
\hline
\end{tabular}

Tabela 3. Variáveis consideradas para a avifauna por área protegida, riqueza, número de espécies ameaçadas de extinção, espécies restritas ao Domínio da Mata Atlântica e espécies restritas ao Domínio do Cerrado.

Table 3. Variables considered for avifauna by protected area, species richness, number of threatened species, species restricted to Atlantic Forest Domain and to Cerrado Domain.

\begin{tabular}{lcccc}
\hline & Riqueza & Espécies Ameaçadas & Mata Atlântica & Cerrado \\
\hline E. Ec. Angatuba & 188 & 3 & 31 & 1 \\
E. Ec. Assis & 136 & 2 & 3 & 2 \\
E. Ec. Avaré & 115 & 0 & 8 & 1 \\
F. E. Batatais & 117 & 5 & 3 & 3 \\
E. Ec. Bauru & 136 & 2 & 5 & 2 \\
E. Ex. Buri & 114 & 2 & 24 & 0 \\
F. E. Cajuru & 131 & 4 & 1 & 4 \\
E. Ec. Itaberá & 111 & 2 & 20 & 0 \\
E. Ec. Itapeva & 94 & 1 & 17 & 0 \\
E. Ex. Itararé & 140 & 3 & 28 & 0 \\
E. Ec. Marília & 125 & 3 & 2 & 2 \\
E. Ec. Paranapanema & 151 & 3 & 19 & 1 \\
E. Ec. Ribeirão Preto & 104 & 2 & 2 & 0 \\
E. Ec. Santa Maria & 120 & 3 & 0 & 5 \\
\hline
\end{tabular}

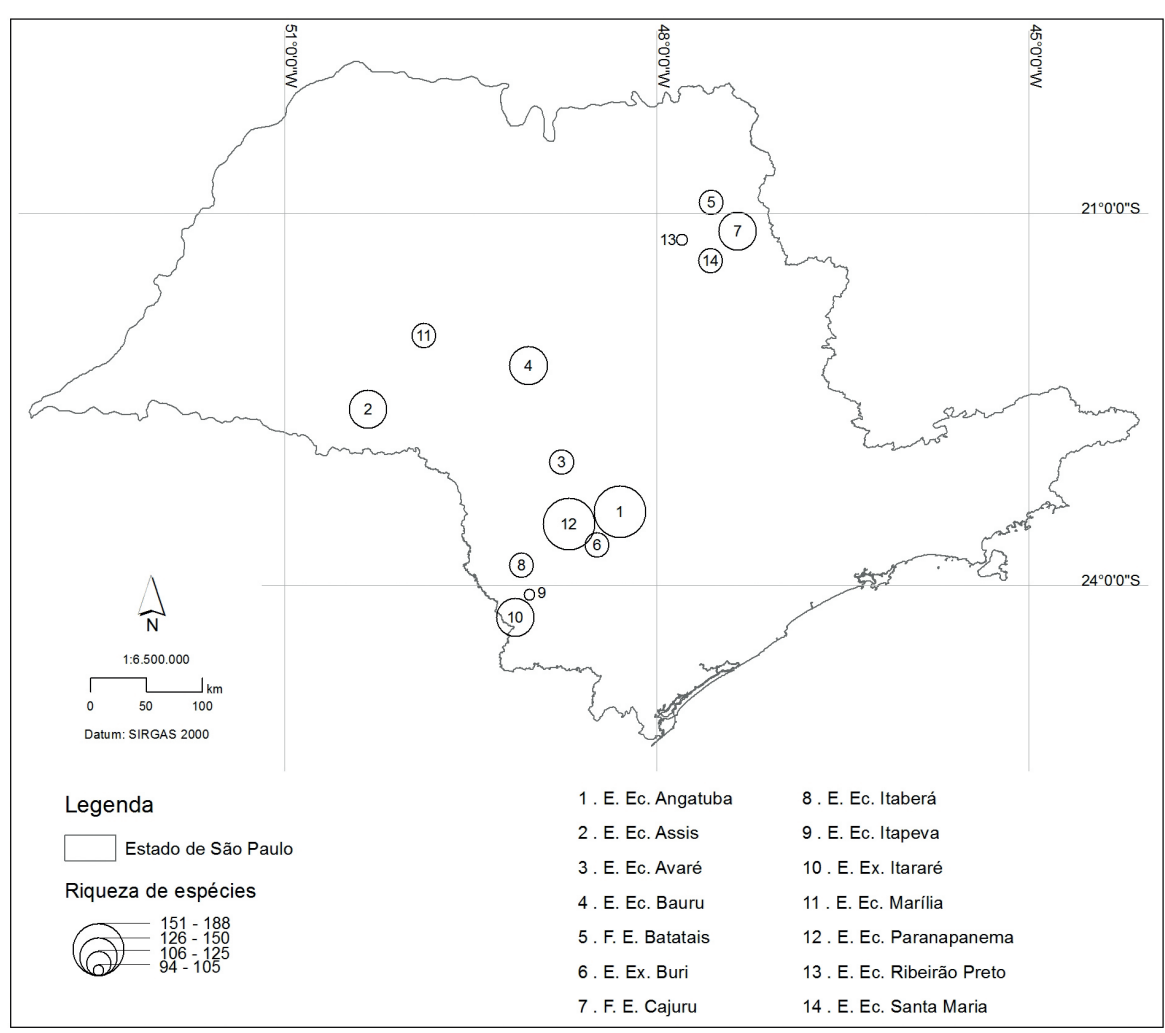

Figura 2. Riqueza de espécies de aves registrados para 14 áreas protegidas do interior do estado de São Paulo. Figure 2. Bird species richness recorded in 14 protected area, in the state of São Paulo, southeastern Brazil.

Rev. Inst. Flor. 28 n. 2 p. $135-157$ dez. 2016 


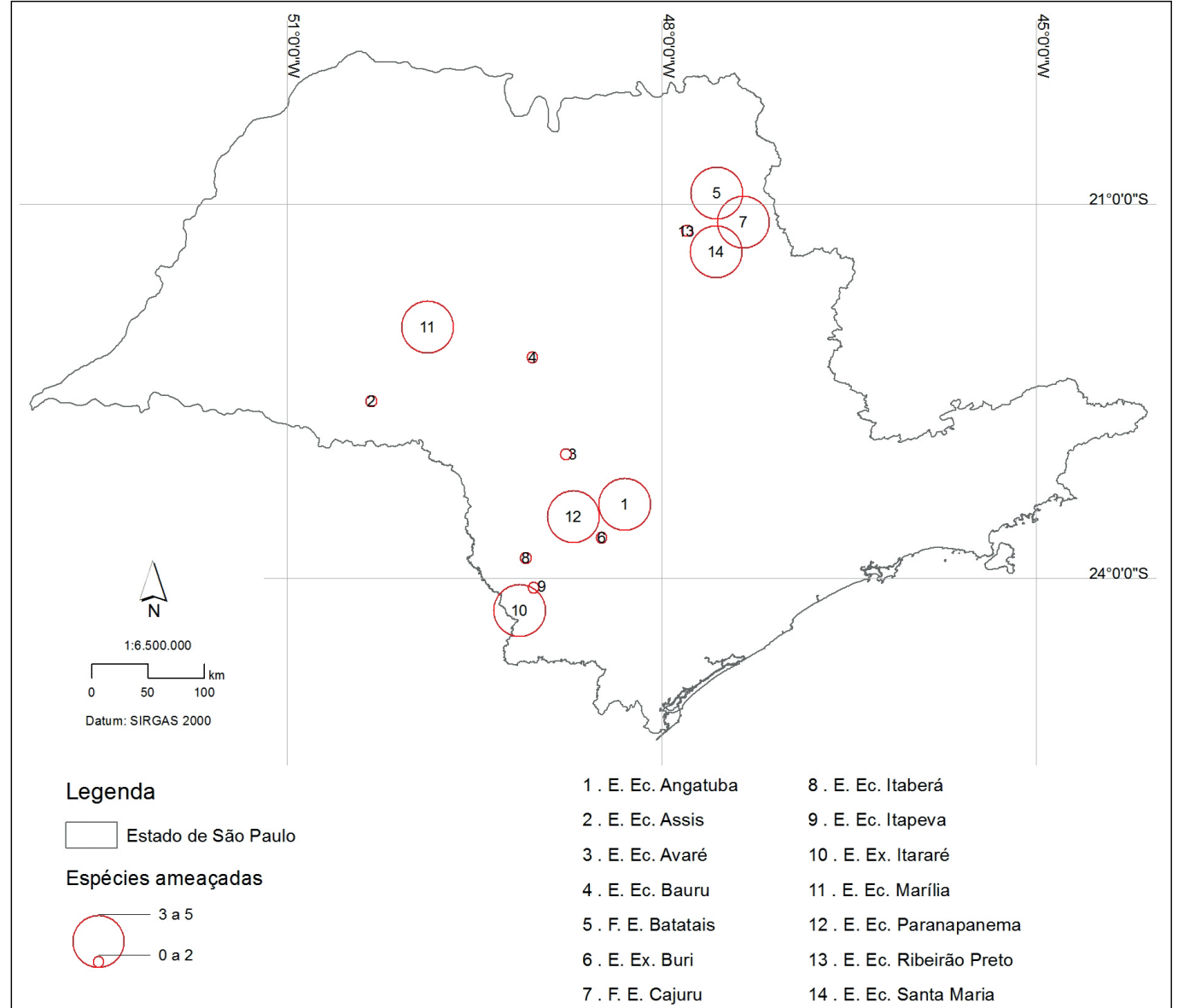

Figura 3. Número de espécies ameaçadas encontrado por área protegida.

Figure 3. Number of threatened bird species recorded in 14 protected areas in the state of São Paulo, southeastern Brazil.

A análise da similaridade na composição as assembleias de aves entre as áreas revelou a existência de dois grandes grupos, formados por localidades ao norte e oeste (Grupo 1) e ao su (Grupo 2) das Cuestas Basálticas (Figuras 4 e 5). Os valores de correlação cofenética variaram entre 8,24 (par Buri-Itapeva) e 11,25 (grupos 1 e 2). No grupo 2, Itapeva é a única área de Cerrado e apresentou maior similaridade com a Floresta Estacional de Buri, apesar de estar geograficamente mais próxima das localidades de Floresta Ombrófila Mista. Itapeva não apresentou espécies de aves exclusivas do Domínio do Cerrado mesmo sua flora sendo característica deste Domínio (Cielo-Filho et al., 2012). No grupo 1 , duas áreas de Cerrado, Cajuru e Santa Maria, presentaram maior similaridade, mas para as demais ocorreram pareamentos entre áreas de

Rev. Inst. Flor. v. 28 n. 2 p. $135-157$ dez. 2016
Cerrado e Floresta Estacional. Silva e Casteleti (2005) consideram as florestas estacionais como um largo cinturão de transição entre as florestas ombrófilas da Mata Atlântica e os domínios adjacentes, destacando que, apesar da escassez de espécies endêmicas, elas são de enorme valor ecológico e evolutivo por possibilitarem interações complexas entre biota com histórias distintas. As áreas florestais de Cerrado não diferiram significativamente quanto à riqueza $(U=22 \mathrm{p}=0,79)$ e nem quanto ao número de espécies ameaçadas encontrado $(\mathrm{U}=15 \mathrm{p}=0,24)$. Também não diferiram quanto ao número médio de espécies restritas à Mata Atlântica $(\mathrm{U}=11,50 \mathrm{p}=0,16)$ ou ao Cerrado $(\mathrm{U}=12$ $\mathrm{p}=0,12)$. Associados à análise de similaridade, estes resultados podem indicar a natureza ecotonal de algumas localidades.

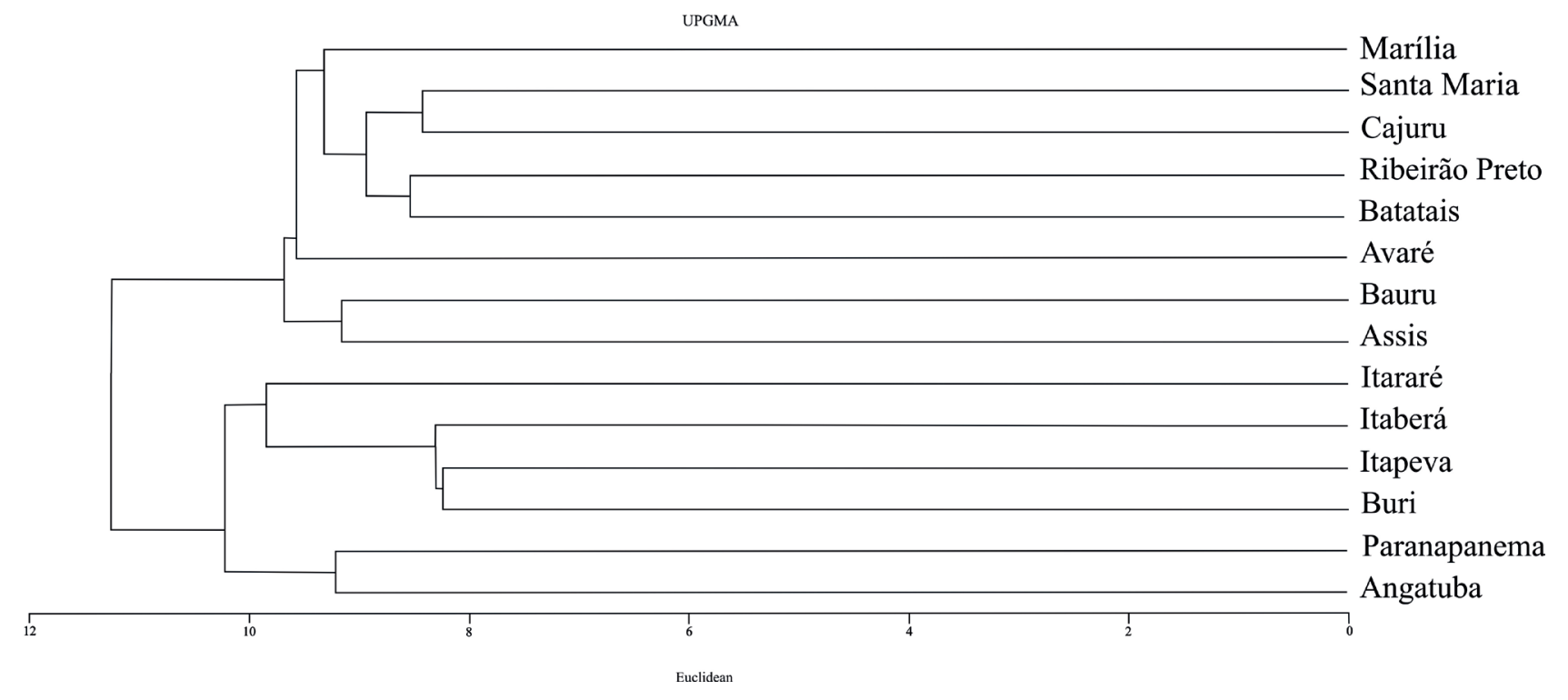

Figura 4. Valores de distância entre a composição da avifauna nas áreas protegidas amostradas, utilizando o método UPGMA e o coeficiente de Distância Euclidiana.

Figure 4. Distance values between bird composition of sampled protected areas in the state of São Paulo, southeastern

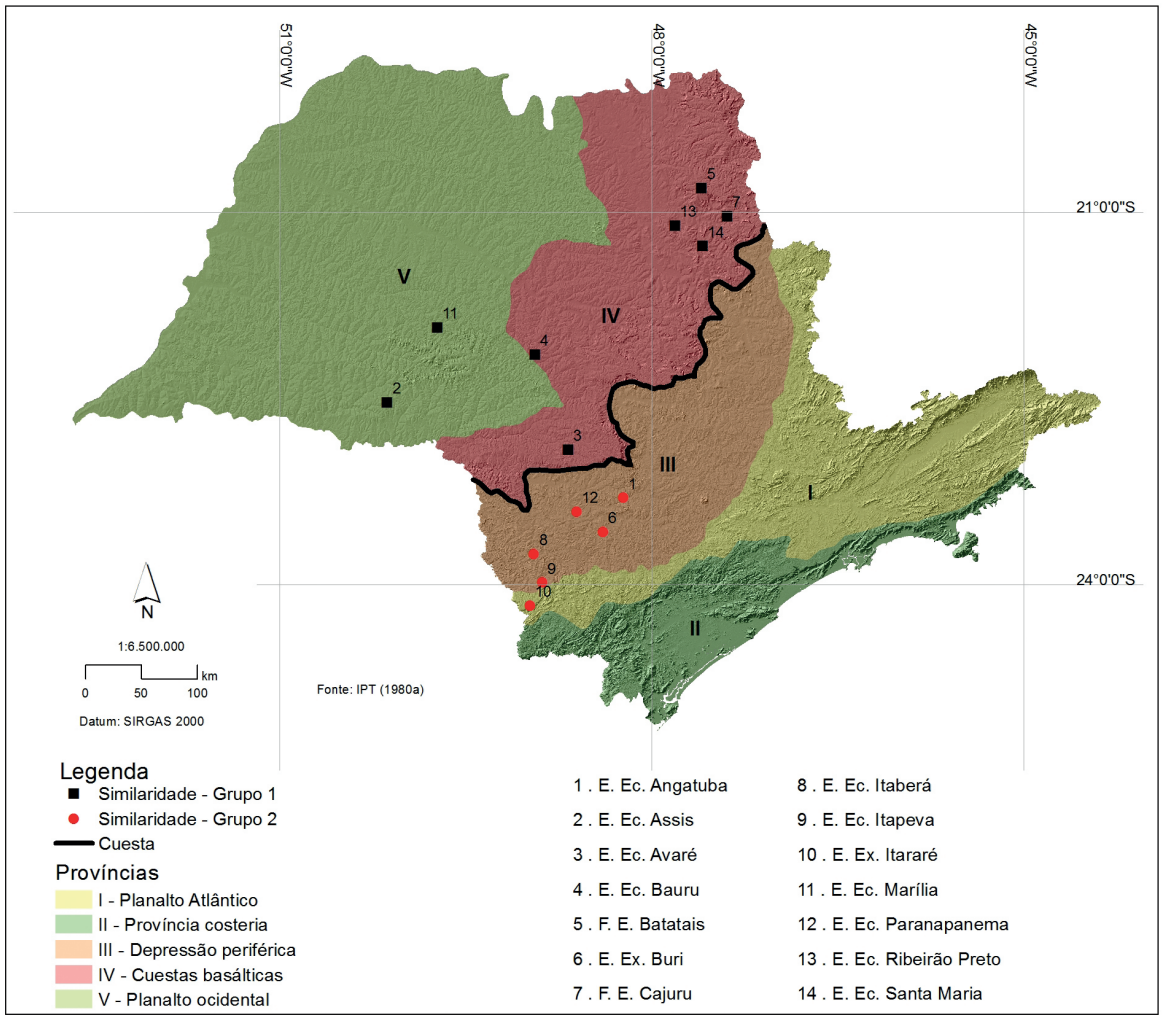

Figura 5. Distribuição das áreas amostradas em relação aos grupos principais quanto à similaridade na composição de espécies de aves.

Figure 5. Distribution of sampled areas in the state of São Paulo, southeastern Brazil, in relation to the main groups on the similarity in the composition of bird species.

Rev. Inst. Flor. v. 28 n. 2 p. $135-157$ dez. 2016 
Entre as variáveis independentes, 0 esforço amostral parece ser o principal fator influenciar nos valores de riqueza e de espécies ameaçadas encontrados por área, pois está diretamente associado à probabilidade de registrar espécies discretas e/ou que ocorrem em baixas densidades (Tabela 4). Nos caso de espécies ameaçadas e restritas ao Cerrado, o número de fitofisionomias presentes também parece ser relevante. Provavelmente, por ser constituído por três biomas distintos (Batalha et al., 2010), o Cerrado favorece maior especificidade de habitat em suas espécies restritas em relação às típicas de Mata Atlântica. Assim, quanto maior o número de fitofisionomias presentes maior chance de detecção de espécies restritas ao Cerrado. Santa Maria, a localidade que apresentou cinco das seis espécies restritas, evidencia este fator, já que Antilophia galeata (Lichtenstein, 1823) e

Myiothlypis leucophrys (Pelzeln, 1868) ocorrem exclusivamente nas matas ciliares, Saltatricula atricollis (Vieillot, 1817) é do cerrado típico, Gubernetes yetapa (Vieillot, 1818) está restrit a brejos com vegetação herbáceo-arbustiva Cyanocorax cristatellus (Temminck, 1823) generalista, sendo assinalada em cerrados típicos, cerradões e áreas antropizadas com arbustos e árvores isoladas. Cabe destacar que metade dessas espécies, consideradas restritas ao Cerrado, na verdade são formas florestais. Além das citada anteriormente, Herpsilochmus longirostris Pelzeln, 1868 ocorre em matas ciliares, matas decíduas e cerradões (Willis e Oniki, 2003). Silva e Santos (2005) consideraram a avifauna do Cerrado como sendo uma avifauna predominantemente florestal, vivendo em um domínio coberto por savanas, já que $72 \%$ das espécies utilizam fitofisionomias florestais.

Tabela 4. Correlação entre as variáveis independentes (colunas) e dependentes (linhas), para a avifauna de 14 áreas protegidas no interior do estado de São Paulo.

Table 4. Correlation between the independent variables (columns) and dependent variables (lines) to avifauna of 14 protected areas in the state of São Paulo, southeastern Brazil.

\begin{tabular}{llll}
\hline & Área (ha) & Número de Fitofisionomias & Esforço Amostral (h) \\
\hline Riqueza & $\mathrm{rs}=-0,24 \mathrm{p}=0,39$ & $\mathrm{rs}=0,41 \mathrm{p}=0,14$ & $\mathrm{rs}=0,61 \mathrm{p}=0,019$ \\
Número de Espécies Ameaçadas & $\mathrm{rs}=-0,09 \mathrm{p}=0,73$ & $\mathrm{rs}=0,55 \mathrm{p}=0,041$ & $\mathrm{rs}=0,75 \mathrm{p}=0,0018$ \\
Número de Espécies Restritas à Mata Atlântica & $\mathrm{rs}=0,03 \mathrm{p}=0,91$ & $\mathrm{rs}=-0,12 \mathrm{p}=0,66$ & $\mathrm{rs}=-0,016 \mathrm{p}=0,95$ \\
Número de Espécies Restritas ao Cerrado & $\mathrm{rs}=-0,21 \mathrm{p}=0,46$ & $\mathrm{rs}=0,55 \mathrm{p}=0,041$ & $\mathrm{rs}=0,49 \mathrm{p}=0,07$ \\
\hline
\end{tabular}

As espécies restritas ao Domínio Mata Atlântica ocorrem no interior ou na borda de florestas, com exceção de Knipolegu. nigerrimus (Vieillot, 1818) que é restrita aos campos de altitude e rupestres (Willis e Oniki, 2003). Leptasthenura setaria (Temminck, 1824) é dependente da presença do pinheiro-brasileiro Araucaria angustifolia (Bertol.) Kuntze, nativo ou cultivado (Antunes et al., 2007). Ribeiro et al. (2013) consideram que para Itaberá a classificação mais adequada seja a de Floresta em Transição entre Floresta Ombrófila Densa e Floresta

. $20.135-157$ dez 2016
A riqueza local apresentou baixa correlação com o número de espécies ameaçada ( $r s=0,50 p=0,06$ ), com o número de espécies com distribuição restrita ao Domínio da Mata Atlântica (rs $=0,21 \mathrm{p}=0,46)$ e com o número de espécies com distribuição restrita ao Domínio do Cerrado ( $r s=0,36 p=0,20)$. O número de espécies com distribuição restrita ao Domínio da Mata Attântic apresentou baixa correlação com o número de espécies ameaçadas ( $r s=-0,22 p=0,44)$ e esteve negativamente correlacionado com o número de espécies restritas ao Domínio do Cerrado (rs $=-0,69$ $\mathrm{p}=0,006$ ).

Remsen (1994) indicou os principais problemas em comparar a riqueza de aves entre áreas com esforços amostrais distintos e com tipo fitofisionômicos diferentes. A comparação entre os valores de riqueza obtidos no presente estudo só seria possível com a padronização do esforço amostral. Outro fator importante é a variação sazonal na detecção de espécies migratórias, as área foram visitadas em períodos distintos do ano Contudo, mesmo considerando estas variáveis, as Estações Ecológicas amostradas não diferira significativamente das outras quatro áreas en relação ao número de espécies ameaçadas de extinção $(\mathrm{U}=8 \mathrm{p}=0,08)$, restritas ao Domínio da Mata Atlântica $(U=17,50 \mathrm{p}=0,72)$ e restrita ao Cerrado $(\mathrm{U}=19 \mathrm{p}=0,88)$.

\section{CONCLUSÕES}

Somadas, as áreas protegidas estudada representam apenas $0,056 \%$ da superfície continental do estado de São Paulo, $139 \mathrm{~km}^{2}$ de $248.209 \mathrm{~km}^{2}$. Como dito anteriormente, boa parte de sua vegetação está em regeneração e efetuamo apenas levantamentos expeditos da avifauna Surpreendentemente, a riqueza encontrad corresponde a $41 \%$ do total de 824 espécies de aves registradas no estado de São Paulo (Figueiredo, 2016), incluindo $12 \%$ do total de 171 espécies ameaçadas (São Paulo, 2014).
As Estações Experimentais e Florestas Estaduais amostradas apresentaram valores similares aos obtidos para as Estações Ecológicas em relação ao número de espécies ameaçadas de extinção, restritas ao Domínio da Mata Atlântica e restritas ao Domínio do Cerrado. Consideramos que em relação à preservação das aves isto justificaria a recategorização das Florestas Estaduais de Batatais e Cajuru e das Estações Experimentais de Buri e Itararé em unidades de conservação mais restritivas como são as Estações Ecológicas

\section{AGRADECIMENTOS}

Aos gestores das Unidades de Conservação amostradas pelo apoio logístico; à Comissão Técnico-Científica do Instituto Florestal po autorizar a execução do projeto, Processo SMA 1516736-008.500/0, e aos dois revisores anônimos pelas críticas e sugestões.

\section{REFERÊNCIAS BIBLIOGRÁFICAS}

ANTUNES, A.Z. Alterações na composição da comunidade de aves ao longo do tempo em um fragmento florestal no sudeste do Brasil. Ararajuba, v. 13, n. 1, p. 47-61, 2005.

. et al. Distribuição de Leptasthenura setaria (Temminck, 1824) (Aves: Furnariidae) no Estado de São Paulo. Biota Neotropica, v. 7, n. 1, 2007. Disponível em: \http://www.biotaneotropica.org.br/v7nl> Acesso em: 16 maio 2016.

AYRES, M. et al. BioEstat 5.0 - aplicações estatísticas nas áreas das ciências biológicas e médicas. Brasília, DF: CNPq, 2010. 290 p.

BATALHA, M.A.; CIANCIARUSO, M.V.; MOTTA-JÚNIOR, J.C. Consequences of simulated loss of open cerrado areas to bird functional diversity. Natureza \& Conservação, v. 8, n. 1, p.34-40, 2010. 
BENCKE, G.A. et al. (Org.). Áreas importantes para a conservação das aves no Brasil: parte I Estados do Domínio da Mata Atlântica. São Paulo: SAVE Brasil, 2006. 494 p.

BIBBY, J.C.; BURGESS, N.D.; HILL, D.A Bird census techniques. London: Academic Press, 1992. $253 \mathrm{p}$

BRASIL. Ministério do Meio Ambiente. Sistema Nacional de Unidades de Conservação da Natureza - SNUC. Lei $\mathbf{n}^{0} \mathbf{9 . 9 8 5}$, de 18 de julho de 2000. Brasília, DF, 2000. 32 p.

CAVARZERE, V.; MORAES, G.P.; DONATELLI, R.J. Avifauna da Estação Ecológica dos Caetetus, interior de São Paulo, Brasil. Papéis Avulsos de Zoologia, v. 49, p. 477-485, 2009.

CIELO-FILHO, R. et al. Floristic aspects of the Itapeva Ecological Station, SP: a protected area in the southern limit of the Cerrado biome. Biota Neotropica, v. 12, n. 2, p. 147-166, 2012.

DEAN, W. A ferro e fogo - a história e a devastação da Mata Atlântica brasileira. São Paulo: Companhia das Letras, 1997. $484 \mathrm{p}$.

DEVELEY, P.F.; CAVANA, D.D.; PIVELLO, V.R Caracterização de grupos biológicos do cerrado Pé-do-Gigante - Aves. In: PIVELLO, V.R. VARANDA, E.M. (Org.). O cerrado Pé-do-Gigante: ecologia e conservação. São Paulo: Secretaria do Meio Ambiente, 2005. p. 121-134.

DIAS, M.M. Avifauna das Estações Ecológica do Jataí e Experimental de Luiz Antônio, São Paulo, Brasil. In: SANTOS, J.E.; PIRES, J.S.R. (Org.)

Estudos integrados em ecossistemas - Estação Ecológica de Jataí. São Carlos: Rima, 2000. v. 1, p. 285-301.

DURIGAN, G. et al. Seleção de fragmentos prioritários para a conservação do Cerrado no Estado de São Paulo. Revista do Instituto Florestal, v. 18, n. 1, p. $23-37,2006$

Rev. Inst. Flor. v. 28 n. 2 p. $135-157$ dez. 2016
FIEKER, C.Z.; REIS M.G.; DIAS-FILHO, M.M. Structure of bird assemblages in dry and seasonally flooded grasslands in Itirapina Ecological Station, São Paulo state. Brazilian Journal of Biology, v. 73, n. 1, p. 91-101, 2013.

FIGUEIREDO, L.F.A. Lista de aves do estado de São Paulo. Versão: 15/1/2016. Disponível en: <www.ceo.org.br>. Acesso em: 2 maio 2016.

FUNDAÇÃO FLORESTAL. Unidades de Conservação. 2016a. Disponível em: $<\mathrm{http}$ ://fflorestal.sp.gov.br/unidades-de-conservacao/>. Acesso em: 6 maio 2016

Planos de Manejo. 2016b. Disponíve em: <http://fflorestal.sp.gov.br/planos-de-manejo/>. Acesso em: 6 maio 2016.

FUNDAÇÃO SOS MATA ATLÂNTICA; NSTITUTO NACIONAL DE PESQUISAS ESPACIAIS. Atlas dos remanescentes florestais da Mata Attântica período 2013 2014 (Relatório Técnico). Disponível em: $<$ http://http://mapas.sosma.org.br/site media/download $<\mathrm{http}: / / \mathrm{http}: / /$ mapas.sosma.org.br/site_media/download/
atlas_20132014_relatorio_tecnico_2015.pdf $>$. Acesso em: 17 nov. 2016.

INSTITUTO CHICO MENDES DE CONSERVAÇÃO DA BIODIVERSIDADE. Unidades de Conservação. Disponível em: $<$ http://www.icmbio.gov.br/portal/biodiversidade/ unidades-de-conservacao/biomas-brasileiros. html>. Acesso em: 17 nov. 2016.

KOVACH, W. L. MVSP - a multi-variate statistica package for Windows, ver. 3.1. Penthraeth: Kovach Computing Services, 1999.

KRONKA, F. J. N. et al. Inventário florestal da vegetação natural do Estado de São Paulo. São Paulo: Secretaria do Meio Ambiente, Instituto Florestal, 2005. 200 p.

LUCINDO, A.S. et al. Birds at Santa Bárbara Ecological Station, one of the last Cerrado remnants in the state of São Paulo, Brazil. Biota Neotropica, v. 15, n. 4, p. 1-15. Disponível em: ‘http:/www.scielo.br/ df/bn/v15n4/1676-0603-bn-1676-0603-2014-0155. pdf.> Acesso em: 6 maio 2016.
MOTTA-JÚNIOR, J.C.; GRANZINOLLI, M.A.M DEVELEY, P.F. Aves da Estação Ecológica de Itirapina, estado de São Paulo, Brasil. Biota Neotropica, v. 8, n. 3, p. 207-227, 2008

PIACENTINI, V.Q. et al. Annotated checklist of the birds of Brazil by the Brazilian Ornithologica Records Committe / Lista comentada das aves do Busl pelo Co / Liste Brileiro de Registos do Bristros v. 23, n. 2, p. 91-298, 2015.

POZZA, D.D.; PIRES, J.R.S. Bird communities in two fragments of semideciduous forest in rura São Paulo state. Brazilian Journal of Biology, v. 63, n. 2 , p. 307-319, 2003.

REMSEN, J.V. Use and misuse of bird lists in community ecology and conservation. Auk, v. 111 n. 1, p. 225-227, 1994.

RIBEIRO, T.M. et al. Mixed rain forest in southeastern Brazil: tree species regeneration an floristic relationships in a remaining stretch of forest near the city of Itaberá, Brazil. Acta Botanica Brasilica, v. 27, n. 1, p. 71-86, 2013.

SÃO PAULO (Estado). Decreto $\mathrm{n}^{\circ} 60.133$ de 7 de fevereiro de 2014. Declara as espécies da fauna silvestre ameaçadas, as quase ameaçadas e as deficientes de dados para avaliação no Estado de São Paulo e dá providencias correlatas. Diário Oficial do Estado de São Paulo, Pode Executivo, v. 124, n. 27, 8 fev. 2014. Seção p. $25-32$

Inventário florestal da vegetação natural do Estado de São Paulo Período 2008-2009. São Paulo: Secretaria do Meio Ambiente, Instituto Florestal Disponível em: $<$ http://www ambiente Fp govbr/sifesp/files/2013/12/ mapainventario.pdf>. Acesso em: 17 nov. 2016

Secretaria do Meio Ambiente. Instituto Florestal. Áreas protegidas. 2016a. Disponíve em: <http://iflorestal.sp.gov.br/areas-protegidas/> Acesso em: 6 maio 2016.

. Secretaria do Meio Ambiente. Instituto Florestal. Planos de Manejo e Gestão. 2016b. Disponível em: <http://iflorestal. sp.gov.br/planos-manejo-gestao/>. Acesso em 6 maio 2016.

Rev. Inst. Flor. v. 28 n. 2 p. $135-157$ dez. 2016
SILVA, J.M.C.; CASTELETI, C.H.M. Estado da biodiversidade da Mata Atlântica brasileira In: GALINDO LEAL, C : CÂMARA, I.G. (Ed.). Mata Atlântica: biodiversidade, ameaças e perspectivas. Belo Horizonte: Conservação Internacional, 2005. p. 43-59.

- SANTOS. M.P.D. A importância relativa dos processos biogeográficos na formação da avifauna do Cerrado e de outros biomas brasileiros. In: SCARIOT, A.; SOUSA-SILVA, J.C.; FELFILI J.M. (Org.). Cerrado: ecologia, biodiversidade e conservação. Brasília, DF: Ministério do Meio Ambiente, 2005. p. 219-233.

TELLES, M.; DIAS, M.M. Bird communities in two fragments of Cerrado in Itirapina, Brazi Brazilian Journal of Biology, v. 70, n. 3 p. $537-550,2010$.

XAVIER, A.F.; BOLZANI, B.M.; JORDÃO S Unidades de conservação da natureza no estado de São Paulo. In: RODRIGUES, R.R.; BONONI, V.L.R. (Org.). Diretrizes para conservação e restauração da biodiversidade no Estado de São Paulo. São Paulo: Instituto de Botânica, 2008 p. $23-42$.

WILLIS, E.O. The composition of avian communities in remanescent woodlots in southern Brazil. Papéis Avulsos de Zoologia, v. 33, p. $1-25,1979$.

ONIKI, Y. Levantamento preliminar de aves em treze áreas do Estado de São Paulo Revista Brasileira de Biologia, v. 41, n. p. 121-135, 1981.

Losses of São Paulo birds are worse in the interior than in Atlantic forests. Ciência e Cultura, v. 44, n. 5, p. 326-328, 1992.

Aves do Estado de São Paulo. Rio Claro: Divisa, 2003. $398 \mathrm{p}$. 